\title{
Reduction of Imines by Hydroxycyclopentadienyl Ruthenium Hydride: Intramolecular Trapping Evidence for Hydride and Proton Transfer Outside the Coordination Sphere of the Metal
}

\author{
Charles P. Casey,* Galina A. Bikzhanova, Qiang Cui, and Ilia A. Guzei \\ Department of Chemistry, University of Wisconsin, Madison, Wisconsin 53706 USA \\ casey@chem.wisc.edu
}

\section{Supporting Information}

General Experimental Information 2

$\left[2,5-\mathrm{Ph}_{2}-3,4-\mathrm{Tol}_{2}\left(\eta^{5}-\mathrm{C}_{4} \mathrm{COH}\right)\right] \mathrm{Ru}(\mathrm{CO})_{2} \mathrm{H}(\mathbf{2}) \quad 2$

$\left[2,5-\mathrm{Ph}_{2}-3,4-\mathrm{Tol}_{2}\left(\eta^{4}-\mathrm{C}_{4} \mathrm{CO}\right)\right](\mathrm{CO})_{2} \mathrm{RuNH}_{2} \mathrm{Ph}(7)$

$\left[2,5-\mathrm{Ph}_{2}-3,4-\mathrm{Tol}_{2}\left(\eta^{4}-\mathrm{C}_{4} \mathrm{CO}\right)\right](\mathrm{CO})_{2} \mathrm{RuNH}_{2} \mathrm{C}_{6} \mathrm{H}_{4}-\mathrm{p}-\mathrm{CHMe}_{2}(\mathbf{1 0})$

Kinetics of Amine Exchange Reaction 3

$\begin{array}{ll}T_{1} \text { measurement of } 2 & 4\end{array}$

DFT Calculations

X-ray of $\left[2,5-\mathrm{Ph}_{2}-3,4-\mathrm{Tol}_{2}\left(\eta^{4}-\mathrm{C}_{4} \mathrm{CO}\right)\right](\mathrm{CO})_{2} \mathrm{RuNH}_{2} \mathrm{C}_{6} \mathrm{H}_{4}-p-\mathrm{NHCH}_{2} \mathrm{Ph}(\mathbf{9})$ 
General Experimental Information. All air-sensitive materials were manipulated under dry nitrogen in a glovebox or by standard high vacuum and Schlenk line techniques. Solvents were dried and distilled under nitrogen prior to use. $\mathrm{N}$ benzylidenemethylamine, $N$-benzylideneaniline, $N$-methylbenzylamine, $N$ phenylbenzylamine, isopropylamine, aniline and $p$-isopropylaniline were used as obtained (Aldrich). 4-Amino- $N$-(benzylidene)aniline ${ }^{1}$ and 4-( $N$-benzylamino)aniline ${ }^{2}$ were prepared as described previously. $\left\{\left[2,5-\mathrm{Ph}_{2}-3,4-\mathrm{Tol}_{2}\left(\eta^{5}-\mathrm{C}_{4} \mathrm{CO}\right)\right]_{2} \mathrm{H}\right\} \mathrm{Ru}_{2}(\mathrm{CO})_{4}(\mu-\mathrm{H})$ (1) ${ }^{3}\left[2,5-\mathrm{Ph}_{2}-3,4-\mathrm{Tol}_{2}\left(\eta^{5}-\mathrm{C}_{4} \mathrm{COH}\right)\right] \mathrm{Ru}(\mathrm{CO})_{2} \mathrm{H}(\mathbf{2}),{ }^{3}\left[2,5-\mathrm{Ph}_{2}-3,4-\mathrm{Tol}_{2}\left(\eta^{4}-\mathrm{C}_{4} \mathrm{CO}\right) \mathrm{Ru}(\mathrm{CO})_{2}\right]_{2}$ $(3),{ }^{3}$ and $\left[2,5-\mathrm{Ph}_{2}-3,4-\mathrm{Tol}_{2}\left(\eta^{4}-\mathrm{C}_{4} \mathrm{CO}\right)\right](\mathrm{CO})_{2} \mathrm{RuNH}_{2}\left(\mathrm{CHMe}_{2}\right) \quad(\mathbf{5})^{4}$ were synthesized according to literature procedures.

${ }^{1} \mathrm{H}$ and ${ }^{13} \mathrm{C}\{\mathrm{H}\}$ NMR spectra were obtained on a Varian Unity 500 spectrometer. Solution IR spectra were measured on a Mattson Polaris FT-IR spectrometer using a 0.1 $\mathrm{mm} \mathrm{CaF}_{2}$ cell.

$\left[2,5-\mathrm{Ph}_{2}-3,4-\mathrm{Tol}_{2}\left(\eta^{5}-\mathrm{C}_{4} \mathrm{COH}\right)\right] \mathbf{R u}(\mathrm{CO})_{2} \mathrm{H}(\mathbf{2}){ }^{3} \quad$ A solution of $\mathbf{1}(11.4 \mathrm{mg}, 0.01$ mmol) in THF $(0.45 \mathrm{~mL})$ in a resealable NMR tube was degassed by three successive freeze-pump-thaw cycles and placed under $1 \mathrm{~atm} \mathrm{H}_{2}$ at $-78{ }^{\circ} \mathrm{C}$. The tube was sealed at $-78{ }^{\circ} \mathrm{C}$ and heated at $90{ }^{\circ} \mathrm{C}$ in a constant temperature bath for $8 \mathrm{~h}$ to form 2 . The THF solvent was evaporated under vacuum and toluene- $d_{8}(0.5 \mathrm{~mL})$ was added.

$\left[\mathbf{2 , 5}-\mathrm{Ph}_{2}-\mathbf{3 , 4}-\mathrm{Tol}_{2}\left(\eta^{4}-\mathrm{C}_{4} \mathbf{C O}\right)\right](\mathbf{C O})_{2} \mathbf{R u N H}_{2} \mathbf{P h}(\mathbf{7})$. Aniline $(7.3 \mu \mathrm{L}, 0.08 \mathrm{mmol})$ was added via syringe to a $\mathrm{CD}_{2} \mathrm{Cl}_{2}$ suspension of ruthenium cyclopentadienone dimer $\mathbf{3}$ (45.6 $\mathrm{mg}, 0.04 \mathrm{mmol}$ ) and the mixture was stirred for $30 \mathrm{~min}$, until all the material dissolved. Solvent was evaporated under vacuum to afford 7 (45.4 mg, $85 \%)$, mp 166-169 ${ }^{\circ} \mathrm{C}(\mathrm{dec}) . \quad \mathrm{IR}\left(\mathrm{CD}_{2} \mathrm{Cl}_{2}\right) 2017$ (s), $1958(\mathrm{~s}) \mathrm{cm}^{-1} .{ }^{1} \mathrm{H} \mathrm{NMR}\left(\mathrm{CD}_{2} \mathrm{Cl}_{2}, 500 \mathrm{MHz}\right) \delta$ 
$2.22\left(\mathrm{~s}\right.$, tolyl $\left.\mathrm{CH}_{3}\right), 4.46\left(\mathrm{~s}, \mathrm{NH}_{2}\right), 6.55\left(\mathrm{~d},{ }^{3} \mathrm{~J}=8.0 \mathrm{~Hz}, 2 \mathrm{H}\right.$, aromatic), $6.92(\mathrm{~m}, 5 \mathrm{H}$, aromatic), $6.99\left(\mathrm{t},{ }^{3} \mathrm{~J}=8.0 \mathrm{~Hz}, 2 \mathrm{H}\right.$, aromatic), $7.03\left(\mathrm{~d},{ }^{3} J=8.0 \mathrm{~Hz}, 4 \mathrm{H}\right.$, aromatic), 7.19 (m, $6 \mathrm{H}$, aromatic), $7.53\left(\mathrm{~m}, 4 \mathrm{H}\right.$, aromatic). ${ }^{13} \mathrm{C}\left\{{ }^{1} \mathrm{H}\right\} \mathrm{NMR}\left(\mathrm{CD}_{2} \mathrm{Cl}_{2}, 125 \mathrm{MHz}\right) \delta 21.17$ (tolyl $\mathrm{CH}_{3}$ ); 84.47 (C 3, 4 of Cp); 103.61 (C 2, 5 of Cp); 118.97, 124.86, 126.81, 128.06, $128.62,128.87,129.43,131.01,132.20,133.09,138.00,146.97$ (aromatic); 163.84 (C1 of $\mathrm{Cp}$ ); 200.06 (CO). HRMS (ESI) $(\mathrm{M}+\mathrm{H})^{+} \mathrm{Calcd}$ for $\mathrm{C}_{39} \mathrm{H}_{31} \mathrm{NO}_{3}{ }^{102} \mathrm{Ru}, 664.1425$; Found, 664.1401.

$\left[2,5-\mathrm{Ph}_{2}-3,4-\mathrm{Tol}_{2}\left(\eta^{4}-\mathrm{C}_{4} \mathrm{CO}\right)\right](\mathrm{CO})_{2} \mathrm{RuNH}_{2} \mathrm{C}_{6} \mathrm{H}_{4}-\mathrm{p}-\mathrm{CHMe}_{2}$ ( 10 ) $p$-Isopropylaniline (12 $\mu \mathrm{L}, 0.08 \mathrm{mmol}$ ) was added via syringe to a $\mathrm{CD}_{2} \mathrm{Cl}_{2}$ suspension of ruthenium cyclopentadienone dimer $\mathbf{3}(50 \mathrm{mg}, 0.04 \mathrm{mmol})$ and the mixture was stirred for $30 \mathrm{~min}$, until all the material dissolved. Solvent was evaporated under vacuum to afford 10 (48 mg, $77 \%$ ), mp 183-186 ${ }^{\circ} \mathrm{C}$ (dec). IR $\left(\mathrm{CD}_{2} \mathrm{Cl}_{2}\right) 2016$ (s), $1957(\mathrm{~s}) \mathrm{cm}^{-1} \cdot{ }^{1} \mathrm{H}$ $\operatorname{NMR}\left(\mathrm{C}_{6} \mathrm{D}_{6}, 500 \mathrm{MHz}\right) \delta 1.78\left(\mathrm{~s}, 6 \mathrm{H}\right.$, tolyl $\left.\mathrm{CH}_{3}\right), 3.06\left(\mathrm{t},{ }^{3} J=5.8 \mathrm{~Hz}, \mathrm{NH}\right), 3.69\left(\mathrm{~d},{ }^{3} J=\right.$ $\left.5.5 \mathrm{~Hz}, \mathrm{CH}_{2}\right), 3.75\left(\mathrm{br} \mathrm{s}, \mathrm{NH}_{2}\right), 5.91\left(\mathrm{~d},{ }^{3} J=8.5 \mathrm{~Hz}, 2 \mathrm{H}\right.$, aromatic), $6.18\left(\mathrm{~d},{ }^{3} J=8.5 \mathrm{~Hz}, 2\right.$ $\mathrm{H}$, aromatic), $6.65\left(\mathrm{~d},{ }^{3} \mathrm{~J}=8.0 \mathrm{~Hz}, 4 \mathrm{H}\right.$, aromatic), 6.97-7.12 (m, $11 \mathrm{H}$, aromatic), 7.21 (d, ${ }^{3} J=8.5 \mathrm{~Hz}, 4 \mathrm{H}$, aromatic), $8.14\left(\mathrm{~d},{ }^{3} \mathrm{~J}=8.5 \mathrm{~Hz}, 4 \mathrm{H}\right.$, aromatic). ${ }^{13} \mathrm{C}\left\{{ }^{1} \mathrm{H}\right\} \mathrm{NMR}\left(\mathrm{CD}_{2} \mathrm{Cl}_{2}\right.$, $125 \mathrm{MHz}$ ) $\delta 21.16$ (tolyl $\mathrm{CH}_{3}$ ); 24.01, 33.73, 84.44 (C 3, 4 of Cp); 103.45 (C 2, 5 of Cp); $118.90,126.75,127.18,128.02,128.59,128.94,131.05,132.21,133.13,137.93,144.56$, 145.61 (aromatic); 163.88 (C1 of $\mathrm{Cp}) ; 200.14(\mathrm{CO})$. $\mathrm{MS}(\mathrm{ESI})(\mathrm{M}+\mathrm{H})^{+}$Calcd for $\mathrm{C}_{42} \mathrm{H}_{37} \mathrm{NO}_{3} \mathrm{Ru}, 706.1895$; Found, 706.1910.

Kinetics of Amine Exchange Reaction will be illustrated for the reaction of aniline with 6 which leads to displacement of $N$-phenylbenzylamine. A solution of aniline (12 mL, $132 \mathrm{mmol})$ in $30 \mathrm{~mL} \mathrm{CD} \mathrm{Cl}_{2}$ was added via a gas-tight syringe to a 
resealable NMR tube containing $6(5.0 \mathrm{mg}, 6.6 \mathrm{mmol}, 0.02 \mathrm{M})$ in $\mathrm{CD}_{2} \mathrm{Cl}_{2}$ at $-40{ }^{\circ} \mathrm{C}$. The tube was evacuated and nitrogen was readded. The tube was inserted into a NMR spectrometer having a probe temperature of $25^{\circ} \mathrm{C}$. The disappearance of 6 was measured by integrating its tolyl methyl resonances at $\delta 2.18$ and 2.24. The formation of free $N$-phenylbenzylamine and 7 was observed. Reactions were followed through three half-lives. All the kinetic runs were done with at least a 10-fold excess of aniline and followed pseudo-first order kinetics.

$T_{1}$ Measurement of $2-\mathrm{H}_{2} \mathrm{NCHMe}_{2}$. At $-60{ }^{\circ} \mathrm{C}$, an inversion-recovery NMR experiment was carried out. An inversion-recovery experiment is a two pulse sequence that involves first a $180^{\circ}$ pulse then a relaxation time (varied) then a $90^{\circ}$ pulse and acquisition of a FID. The proton resonances height versus relaxation time were fit to an exponential fitting program provided by Varian software to determine the $T_{1}$ times. 
DFT Computational methods. The model reactions of $\left(\mathrm{C}_{5} \mathrm{H}_{4} \mathrm{OH}\right) \mathrm{Ru}(\mathrm{CO})_{2} \mathrm{H}$ (2-H) with both $\mathrm{H}_{2} \mathrm{C}=\mathrm{O}$ and $\mathrm{H}_{2} \mathrm{C}=\mathrm{NCH}_{3}$ were calculated. The geometries for all critical species (reactants, intermediates, transition states and products) were optimized using the hybrid density functional theory, B3LYP. ${ }^{5}$ The effective core potential (ECP) of Hay and $\mathrm{Wadt}^{6}$ and the corresponding basis set (augmented by an $f$ function) were used for $\mathrm{Ru}$; the $6-31++\mathrm{G}(\mathrm{d}, \mathrm{p})$ basis $^{7}$ was used for all other main group elements. Vibrational frequency calculations were subsequently carried out to verify the character of the optimized structures, and to obtain the zero-point vibrational energies. To estimate the effect of solvation, single point calculations with an implicit solvation model $\left(\right.$ IEF-PCM $\left.{ }^{8}\right)$ were carried out at the gas phase optimized structures; a dielectric constant of 7.58 was used for tetrahydrofuran (THF) as the solvent. Moreover, a complex between THF and $\mathbf{2}-\mathbf{H}$ was computed to estimate the binding energy between THF and the Cp-OH group of $\mathbf{2}-\mathbf{H}$; this was motivated by the consideration that the aldehyde or imine has to replace a THF molecule in the first solvation shell of $\mathbf{2}-\mathbf{H}$ during the reaction. Finally, a set of single point energy calculations at the MP2 level were carried out in the gas phase to obtain better estimates of energetics; a larger basis set, $6-311++\mathrm{G}(\mathrm{d}, \mathrm{p}),{ }^{9}$ was used for the main group elements in these MP2 calculations. All calculations were performed with the Gaussian98 program. ${ }^{10}$ The optimized structures for $\mathrm{H}{ }_{2} \mathrm{C}=\mathrm{O}$ and $\mathrm{H}_{2} \mathrm{C}=\mathrm{NCH}_{3}$ reduction are shown in Figures S1-S4, and the energetics at various levels are summarized in Tables S1-S2 and in reaction energy diagrams shown in Figures S5 and S6.

\section{The mechanism of $\mathrm{H}_{2} \mathrm{C}=\mathrm{O}$ reduction and estimation of its activation}

enthalpy. As shown in Table S1 and Figure S5, the activation enthalpy for reduction of formaldehyde, calculated from the enthalpy difference between $\mathbf{C}-\mathbf{O}$ (formaldehyde 
hydrogen bonded to $\mathrm{CpOH}$ ) and TS-O (transition state for formaldehyde reduction), is $9.1 \mathrm{kcal} \mathrm{mol}^{-1}$ at the MP2 level (including zero-point correction and solvation correction at the B3LYP level). However, we note that the formaldehyde has to replace a THF molecule bound to $\mathbf{2}-\mathbf{H}$ to form $\mathbf{C}-\mathbf{O}$. Calculations show that the binding energy of THF in $\mathbf{2 - H} \cdot \mathbf{T H F}$ is $4.7 \mathrm{kcal} \mathrm{mol}^{-1}$ (including solvation correction) larger than that of formaldehyde in $\mathbf{C - O}$. Therefore, the apparent activation enthalpy for formaldehyde reduction can be estimated to be $4.7+9.1=13.8 \mathrm{kcal} \mathrm{mol}^{-1}$, which is close to the experimental value of $12 \mathrm{kcal} \mathrm{mol}^{-1}$. TS-O can be classified as an early transition state since the $\mathrm{CpO}-\mathrm{H}$ and the $\mathrm{Ru}-\mathrm{H}$ distances are lengthened by only $0.1 \AA$ in going from $\mathbf{C}$ -

\section{O to TS-O.}

Different binding modes of the formaldehyde reduction products to $\left(\mathrm{C}_{5} \mathrm{H}_{4} \mathrm{OH}\right) \mathrm{Ru}(\mathrm{CO})_{2}$. Following the mechanism for reduction of formaldehyde in Figure 4, the direct product of reduction is $\mathbf{D}-\mathbf{O}\left(-10.7 \mathrm{kcal} \mathrm{mol}^{-1}\right)$, which has a hydrogen bond between the $\mathrm{O}-\mathrm{H}$ of methanol and the cyclopentadienone carbonyl and an agostic interaction between a $\mathrm{C}-\mathrm{H}$ bond of methanol and $\mathrm{Ru}$. Breaking the agostic interaction gives intermediate E-O $\left(-2.7 \mathrm{kcal} \mathrm{mol}^{-1}\right)$, which retains the hydrogen bond between the $\mathrm{O}-\mathrm{H}$ of methanol and the cyclopentadienone carbonyl; this implies a strong agostic interaction in D-O of about $8.0 \mathrm{kcal} \mathrm{mol}^{-1}$. Breaking the hydrogen bond gives intermediate $\mathbf{A}-\mathbf{H}\left(+0.2 \mathrm{kcal} \mathrm{mol}^{-1}\right)$, the unsaturated cyclpentadienone ruthenium dicarbonyl intermediate, which has no interaction with methanol; this implies a hydrogen bond strength in $\mathbf{E}-\mathbf{O}$ (and D-O) of about $2.9 \mathrm{kcal} \mathrm{mol}^{-1}$. Coordination of the alcohol to ruthenium gives an alcohol complex F-O $\left(-19.9 \mathrm{kcal} \mathrm{mol}^{-1}\right)$, which has an internal hydrogen bond from the $\mathrm{O}-\mathrm{H}$ of coordinated methanol to the cyclopentadienone 
carbonyl. Combination of ruthenium hydride $\mathbf{2}-\mathbf{H}$ with unsaturated intermediate $\mathbf{A}-\mathbf{H}$ produces the diruthenium bridging hydride $\mathbf{1}-\mathbf{H}\left(-24.8 \mathrm{kcal} \mathrm{mol}^{-1}\right)$. In agreement with the great stability calculated for $\mathbf{1 - H}$, the products of the reduction of aldehydes by $\mathbf{2}$ are alcohol and diruthenium bridging hydride $\mathbf{1}$.

How facile is the conversion of the direct product of reduction D-O, which has both a hydrogen bond and an agostic interaction linking methanol to the Ru complex, to the alcohol complex $\mathbf{F}-\mathbf{O}$ or to the thermodynamic product $\mathbf{1}-\mathbf{H}$ ? Considering the significant structural changes, we only estimated the energy cost associated the isomerization to alcohol complex $\mathbf{F}-\mathbf{O}$ with breaking the agostic interaction between $\mathrm{Ru}$ and the $\mathrm{CH}$ to give $\mathbf{D - O}$. In other words, we assume that the isomerization occurs through breaking the agostic interaction followed by a rapid coordination of the oxygen lone pair to the metal; thus the strength of the agostic interaction (energy difference between $\mathbf{D}-\mathbf{O}$ and $\mathbf{E}-\mathbf{O}=7.0 \mathrm{kcal} \mathrm{mol}^{-1}$ ) gives an estimate for the energy bottleneck of the isomerization. For conversion of $\mathbf{D}-\mathbf{O}$ to the thermodynamic product $\mathbf{1}-\mathbf{H}$, the reaction might involve trapping of $\mathbf{E}-\mathbf{O}$ by metal hydride $\mathbf{2}-\mathbf{H}$ (energy bottleneck = energy difference between $\mathbf{D}-\mathbf{O}$ and $\left.\mathbf{E}-\mathbf{O}=7.0 \mathrm{kcal} \mathrm{mol}^{-1}\right)$. Alternatively, formation of 1-H might involve breaking both the agositic and hydrogen bonding interactions in D-O to form unsaturated intermediate $\mathbf{A}-\mathbf{H}$, which is then trapped by $\mathbf{2}-\mathbf{H}$ (energy bottleneck $=$ energy difference between $\mathbf{D}-\mathbf{O}$ and $\mathbf{A}-\mathbf{H}=10.9 \mathrm{kcal} \mathrm{mol}^{-1}$ ).

The mechanism for reduction of $\mathbf{H}_{2} \mathbf{C}=\mathrm{NCH}_{3}$ (Figures $4 \mathrm{~S}$ and $6 \mathrm{~S}$ and Table S2) is similar to that for reduction of $\mathrm{H}_{2} \mathrm{C}=\mathrm{O}$, but there are significant differences. In contrast to the early transition state seen for $\mathrm{H}_{2} \mathrm{C}=\mathrm{O}$ reduction, the transition state for $\mathrm{H}_{2} \mathrm{C}=\mathrm{NCH}_{3}$ reduction TS-N shows that the $\mathrm{CpO}-\mathrm{H}$ hydrogen has been substantially transferred to $\mathrm{N}$ 
(1.364 $\AA \mathrm{O}---\mathrm{H}, 1.134 \AA \mathrm{H}---\mathrm{N})$ while the $\mathrm{Ru}-\mathrm{H}$ distance lengthened by only $0.1 \AA$ in going from $\mathbf{C}-\mathbf{N}$ to TS-N. The activation enthalpy for reduction of $\mathrm{H}_{2} \mathrm{C}=\mathrm{NCH}_{3}$ calculated from the enthalpy difference between $\mathbf{C}-\mathbf{N}\left(\mathrm{H}_{2} \mathrm{C}=\mathrm{NCH}_{3}\right.$ hydrogen bonded to $\left.\mathrm{CpOH}\right)$ and TS-N (transition state for $\mathrm{H}_{2} \mathrm{C}=\mathrm{NCH}_{3}$ reduction) is $4.8 \mathrm{kcal} \mathrm{mol}^{-1}$ at the MP2 level (including zero-point correction and solvation correction at the B3LYP level). We noted that $\mathrm{H}_{2} \mathrm{C}=\mathrm{NCH}_{3}$ has to replace a THF molecule bound to $\mathbf{2}-\mathbf{H}$ to form $\mathbf{C}-\mathbf{N}$. This is a small difference since calculations show that the binding energy of THF in $\mathbf{2}-\mathbf{H} \cdot \mathbf{T H F}$ is $0.3 \mathrm{kcal} \mathrm{mol}^{-1}$ (including solvation correction) smaller than that of $\mathrm{H}_{2} \mathrm{C}=\mathrm{NCH}_{3}$ in $\mathbf{C}-\mathbf{N}$. The calculated activation enthalpy for $\mathrm{H}_{2} \mathrm{C}=\mathrm{NCH}_{3}$ reduction starting from $\mathbf{2}-\mathbf{H} \cdot \mathbf{T H F}$ (4.8 $-0.2=4.5 \mathrm{kcal} \mathrm{mol}^{-1}$ ) is $9.3 \mathrm{kcal} \mathrm{mol}^{-1}$ lower than that for formaldehyde reduction. This is consistent with the faster rates observed for reaction of $\mathbf{2}$ with imines compared to aldehydes.

\section{Different binding modes of the $\mathrm{H}_{2} \mathrm{C}=\mathrm{NCH}_{3}$ reduction products to} $\left(\mathrm{C}_{5} \mathrm{H}_{4} \mathrm{OH}\right) \mathbf{R u}(\mathrm{CO})_{2}$. Following the mechanism for reduction of $\mathrm{H}_{2} \mathrm{C}=\mathrm{NCH}_{3}$ in Figure 5, the direct product of reduction is $\mathbf{D}-\mathbf{N}\left(-17.3 \mathrm{kcal} \mathrm{mol}^{-1}\right)$, which has a hydrogen bond between the $\mathrm{N}-\mathrm{H}$ of dimethylamine and the cyclopentadienone carbonyl and an agostic interaction between a $\mathrm{C}-\mathrm{H}$ bond of dimethylamine and $\mathrm{Ru}$. Breaking the agostic interaction gives intermediate $\mathbf{E}-\mathbf{N}\left(-4.0 \mathrm{kcal} \mathrm{mol}^{-1}\right)$, which retains the hydrogen bond between the $\mathrm{N}-\mathrm{H}$ of dimethylamine and the cyclopentadienone carbonyl; this implies a strong agostic interaction in $\mathbf{D}-\mathbf{N}$ of about $9.3 \mathrm{kcal} \mathrm{mol}^{-1}$. Breaking the hydrogen bond gives intermediate A-H (-2.2 $\left.\mathrm{kcal} \mathrm{mol}^{-1}\right)$, which has no interaction with methanol; this implies a hydrogen bond strength in $\mathbf{E}-\mathbf{N}$ (and $\mathbf{D}-\mathbf{N}$ ) of about $2.9 \mathrm{kcal} \mathrm{mol}^{-1}$. Coordination of dimethylamine to ruthenium gives amine complex F-N (-41.5 kcal 
$\mathrm{mol}^{-1}$ ), which has an internal hydrogen bond from the $\mathrm{N}-\mathrm{H}$ of coordinated dimethylamine to the cyclopentadienone carbonyl. Combination of ruthenium hydride $\mathbf{2}-\mathbf{H}$ with unsaturated intermediate A-H produces the diruthenium bridging hydride $\mathbf{1 - H}$ (-27.0 $\mathrm{kcal} \mathrm{mol}^{-1}$ ). In contrast to aldehyde reduction by $\mathbf{2}$ whish leads to diruthenium bridging hydride $\mathbf{1}$, imine reduction leads to the formation of more stable amine complexes.

The barrier for conversion of the direct reduction product $\mathbf{D}-\mathbf{N}$, which has both a hydrogen bond and an agostic interaction linking dimethylamine to the Ru complex, to the amine complex $\mathbf{F}-\mathbf{N}$ can be estimated from the energy cost associated with breaking the agostic interaction between $\mathrm{Ru}$ and the $\mathrm{CH}$ to give $\mathbf{D}-\mathbf{N}$, which then rapidly coordinates nitrogen to the metal to give $\mathbf{F}-\mathbf{N}$. The strength of the agostic interaction is estimated from the energy difference between $\mathbf{D}-\mathbf{N}$ and $\mathbf{E}-\mathbf{N}=13.3 \mathrm{kcal} \mathrm{mol}^{-1}$. 
Figure S1. Optimized structures of $\mathbf{2}-\mathbf{H}$ and $\mathbf{2}-\mathbf{H} \cdot \mathbf{T H F}$, and $\mathbf{1}$ at the B3LYP level (see footnote of Table S1). The distances are given in Angstroms, and the angles are in degrees.
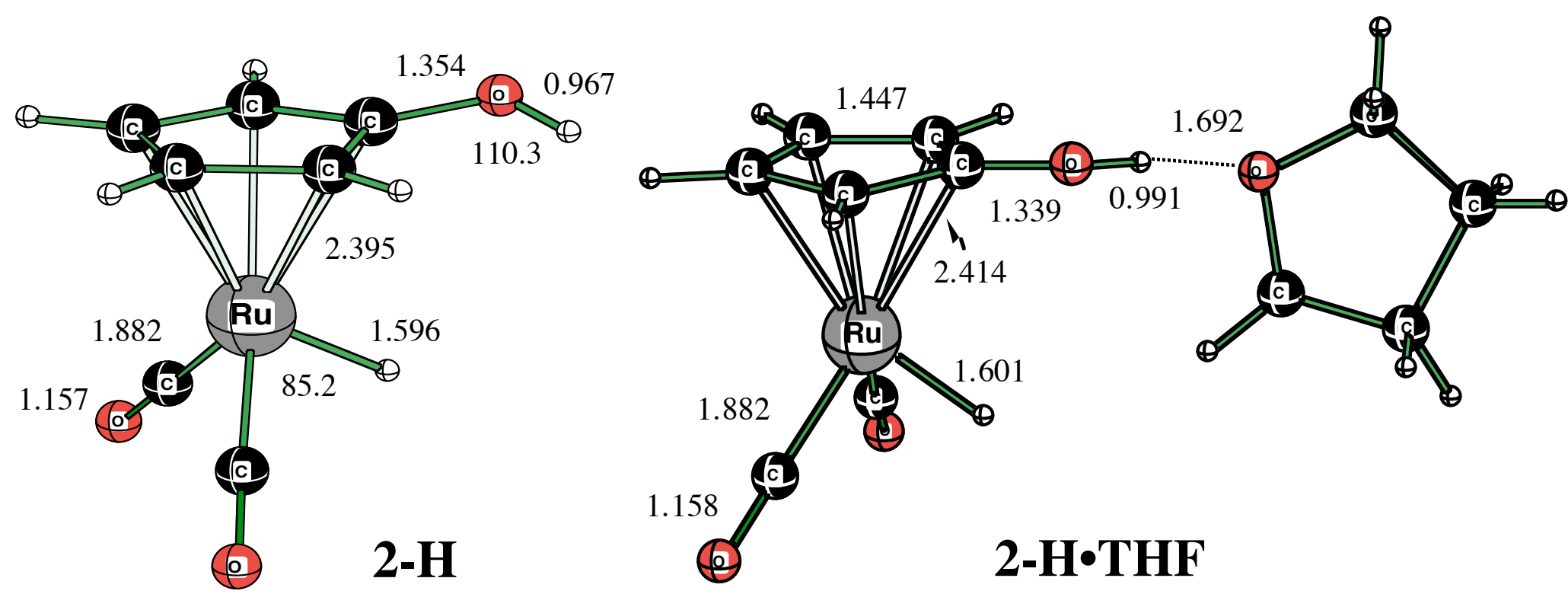
Figure S2. Optimized structure of $\mathbf{1 - H}$ at the B3LYP/BSII+PCM level. The binding energy of $\mathbf{1}-\mathbf{H}$ relative to $\mathbf{2}-\mathbf{H}+\mathbf{A}-\mathbf{H}$ without $\mathrm{ZPE}$ is $-25.0 \mathrm{kcal} \mathrm{mol}^{-1}$. The distances are given in Angstroms, and the angles are in degrees.

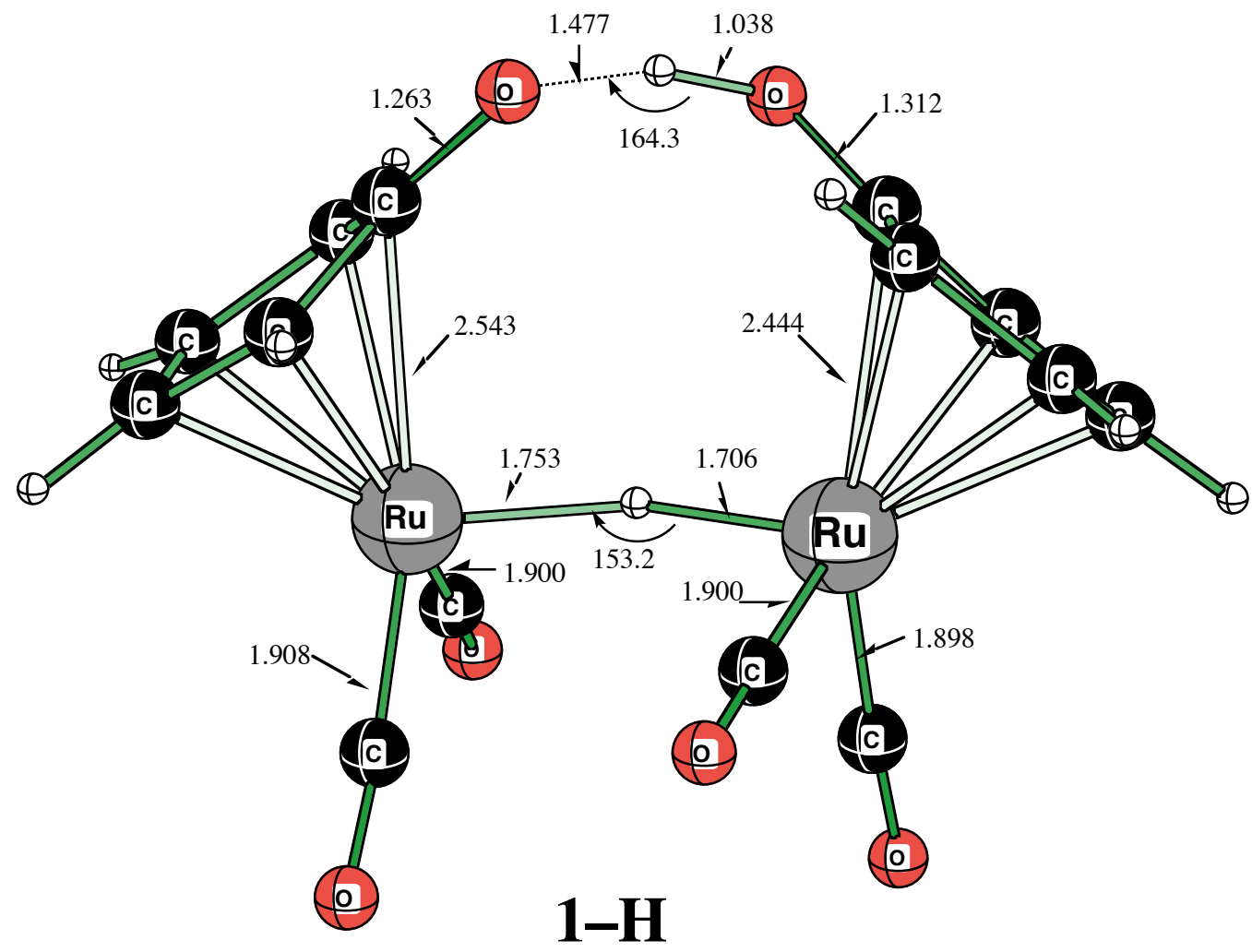


Figure S3. Optimized geometries for critical structures involved in the reduction of formaldehyde by the model compound 2-H (all substituents on the $\mathrm{Cp}$ ring ( $\mathrm{Ph}, \mathrm{Tol})$ were replaced by hydrogens). The structures were optimized at the B3LYP level with doublezeta plus polarization quality basis set (see footnote of Table S1). The distances are given in Angstroms, and the angles are in degrees.
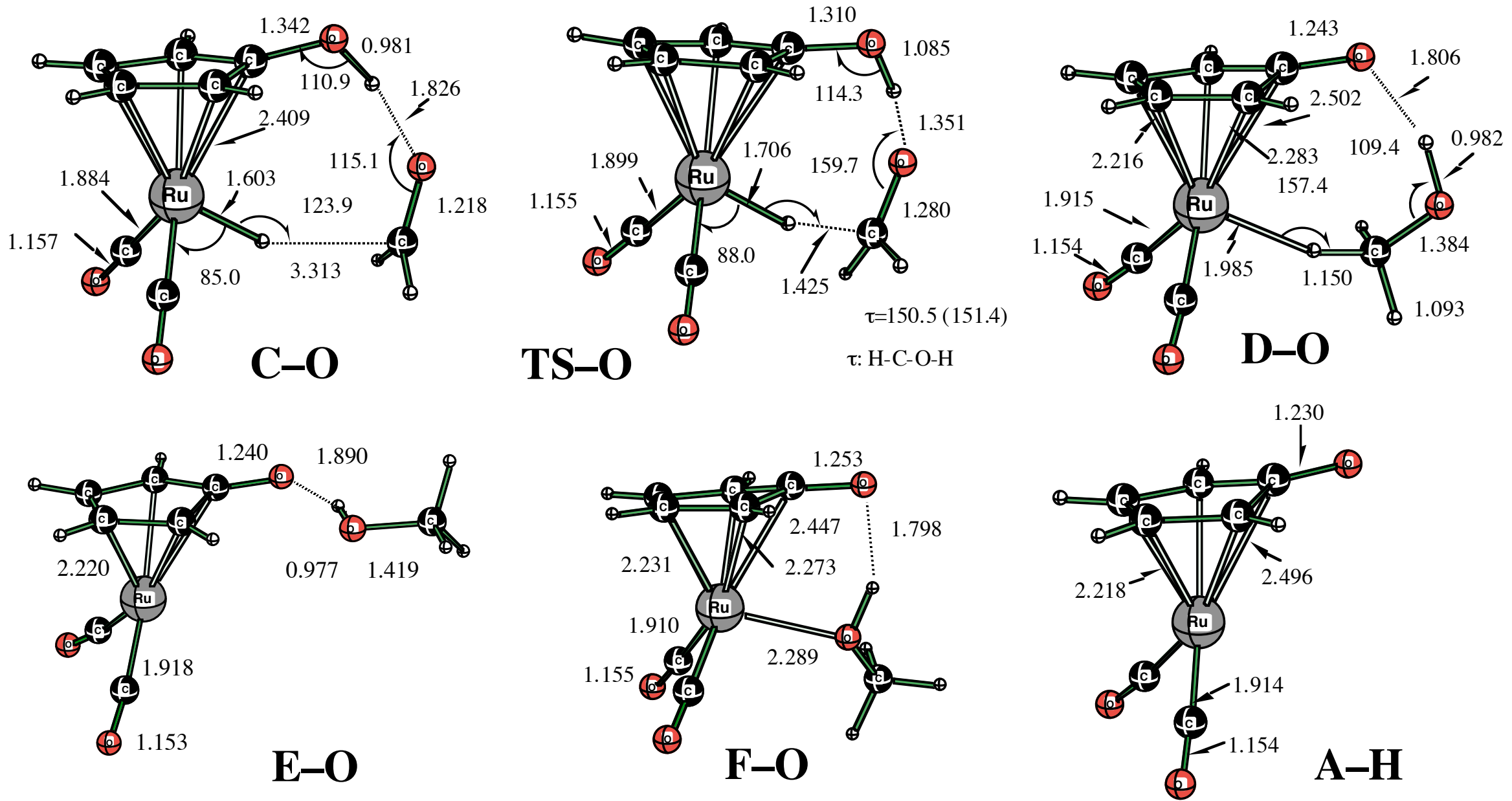
Table S1. Energetics of critical structures (reactant, intermediates, transition states and product) for the reduction of formaldehyde by model $\mathrm{Ru}$ compound $\mathbf{2}-\mathbf{H}$.

\begin{tabular}{lrrr}
\hline & B3LYP/BSII $^{\mathrm{b}}$ & B3LYP/BSII $^{\mathrm{b}}+$ PCM $^{\mathrm{c}}$ & MP2/BSIII $^{\mathrm{b}}+\mathrm{PCM}^{\mathrm{c}}$ \\
\hline $\mathbf{2}-\mathbf{H}$ & -589.98578 & -589.98579 & -588.44754 \\
$\mathrm{H}_{2} \mathrm{C}=\mathrm{O}$ & -114.50977 & -114.51028 & -114.24226 \\
$\mathbf{C}-\mathbf{O}$ & -6.7 & -2.4 & -5.3 \\
$\mathbf{T S}-\mathbf{O}$ & 0.7 & 2.7 & 3.8 \\
$\mathbf{D}-\mathbf{O}$ & -7.6 & -6.3 & -10.7 \\
$\mathbf{A - H}+\mathrm{CH}_{3} \mathrm{OH}$ & 1.3 & -2.0 & 0.2 \\
$\mathbf{E - O}$ & -4.9 & -3.7 & -2.7 \\
$\mathbf{F}-\mathbf{O}$ & -16.5 & -14.9 & -19.9 \\
$\mathbf{2 - H \bullet T H F}+\mathbf{H}_{\mathbf{2}} \mathbf{C}=\mathbf{O}$ & -11.5 & -7.1 & -10.0 \\
\hline
\end{tabular}

a. For reactant molecules (2-H, formaldehyde), the total energies are given in hartree in italics. For all other species, relative energies are given in kcal/mol. Zero-point energy corrections were included for all relative energies. For the $\mathrm{Ru}$ compound $\mathbf{2 - \mathbf { H }}$, all substituents on the $\mathrm{Cp}$ ring $(\mathrm{Ph}, \mathrm{Tol})$ were replaced by hydrogens. For structures, refer to Figures S1-S3.

b. BSII: Hay-Wadt Lanl2dz basis + f polarization function on $\mathrm{Ru}, 6-31++\mathrm{G}(\mathrm{d}, \mathrm{p})$ on all other main group elements. BSIII: Hay-Wadt Lanl2dz basis + f polarization function on $\mathrm{Ru}, 6-$ $311++\mathrm{G}(\mathrm{d}, \mathrm{p})$ on all other main group elements. In the MP2 calculations, geometries optimized at the B3LYP/BSII calculations were used.

c. In PCM calculations, the Pauling set of radii was used; the solvent was THF. PCM calculations were only carried out at the B3LYP/II level at gas phase optimized structures. 
Table S2. Energetics of critical structures (reactant, intermediates, transition states and product) for the reduction of $\mathrm{H}_{2} \mathrm{C}=\mathrm{NCH}_{3}$ by $\mathrm{Ru}$ compound $\mathbf{2}-\mathbf{H}$. ${ }^{\mathrm{a}}$

\begin{tabular}{lrrr}
\hline & B3LYP/BSII & $\begin{array}{r}\text { B3LYP/BSII }^{\mathrm{b}}+ \\
\text { PCM }^{\mathrm{c}}\end{array}$ \\
\hline $\mathbf{2 - H}$ & -589.98578 & -589.98579 & -588.44754 \\
$\mathrm{H}_{2} \mathrm{C}=\mathrm{NCH}_{3}$ & -133.95333 & -133.95507 & -133.57246 \\
$\mathbf{C}-\mathbf{N}$ & -10.3 & -5.6 & -10.3 \\
$\mathbf{T S}-\mathbf{N}$ & -1.6 & 1.5 & -5.5 \\
$\mathbf{D}-\mathbf{N}$ & -10.8 & -7.5 & -17.3 \\
$\mathbf{A - H}+\left(\mathrm{CH}_{3}\right)_{2} \mathrm{NH}$ & -0.2 & -2.8 & -2.2 \\
$\mathbf{E}-\mathbf{N}$ & -3.8 & -0.8 & -4.0 \\
$\mathbf{F}-\mathbf{N}$ & -29.9 & -24.5 & -41.5 \\
$\mathbf{2 - H} \mathbf{H} \mathbf{T H F}+\mathrm{H}_{2} \mathrm{C}=\mathrm{NCH}_{3}$ & -11.5 & -7.1 & -10.0 \\
\hline
\end{tabular}

a. For notations, see footnotes of Table S1. For structures, refer to Figure S1, S-2, and S4. 
Figure S4. Optimized geometries for critical structures involved in the reduction of $\mathrm{CH}_{2}=\mathrm{NCH}_{3}$ by the model compound 2-H (all substituents on the $\mathrm{Cp}$ ring ( $\mathrm{Ph}, \mathrm{Tol})$ were replaced by hydrogens). The structures were optimized at the B3LYP level with double-zeta plus polarization quality basis set (see footnote of Table S1). The distances are given in Angstroms, and the angles are in degrees.
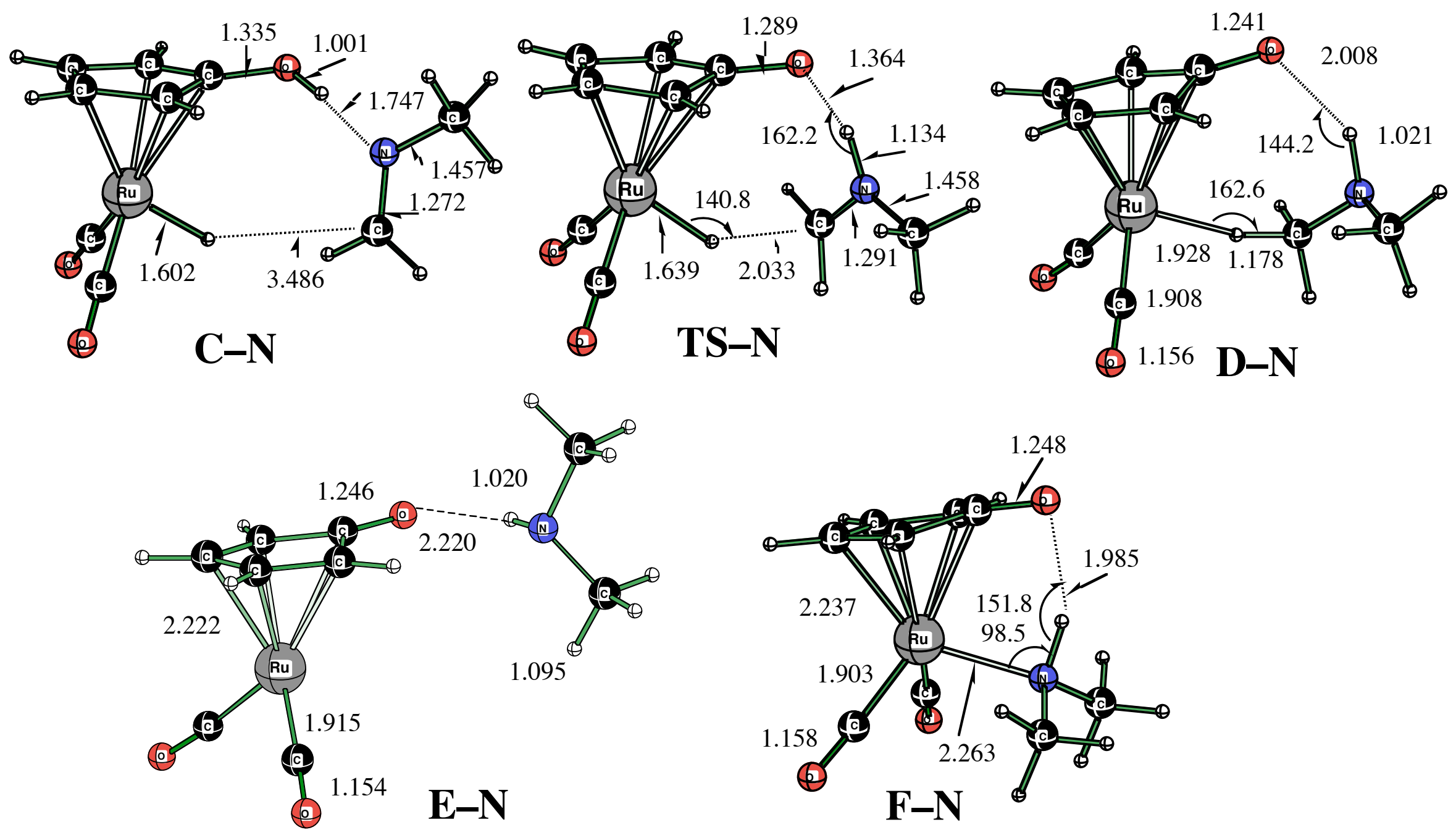
Figure S5. Schematic potential energy surface (based on the MP2 results shown in Table S1) for the reduction of formaldehyde by the model compound $\mathbf{2}-\mathbf{H}$. To be consistent during the entire process, the energy of a THF molecule infinitely apart from the calculated structure is included for all species after isolated reactant and $\mathbf{2}-\mathbf{H}$ (positive horizontal axis). The energy level shown for $\mathbf{1 - H}$ of $\mathbf{- 2 4 . 8}$ kcal mol ${ }^{-1}$ is actually for $\mathbf{1 - H}+\mathrm{CH}_{3} \mathrm{OH}$ relative to $\mathbf{2}-\mathbf{H}+\mathbf{2}-\mathbf{H}+\mathrm{H}_{2} \mathrm{C}=\mathrm{O}$.

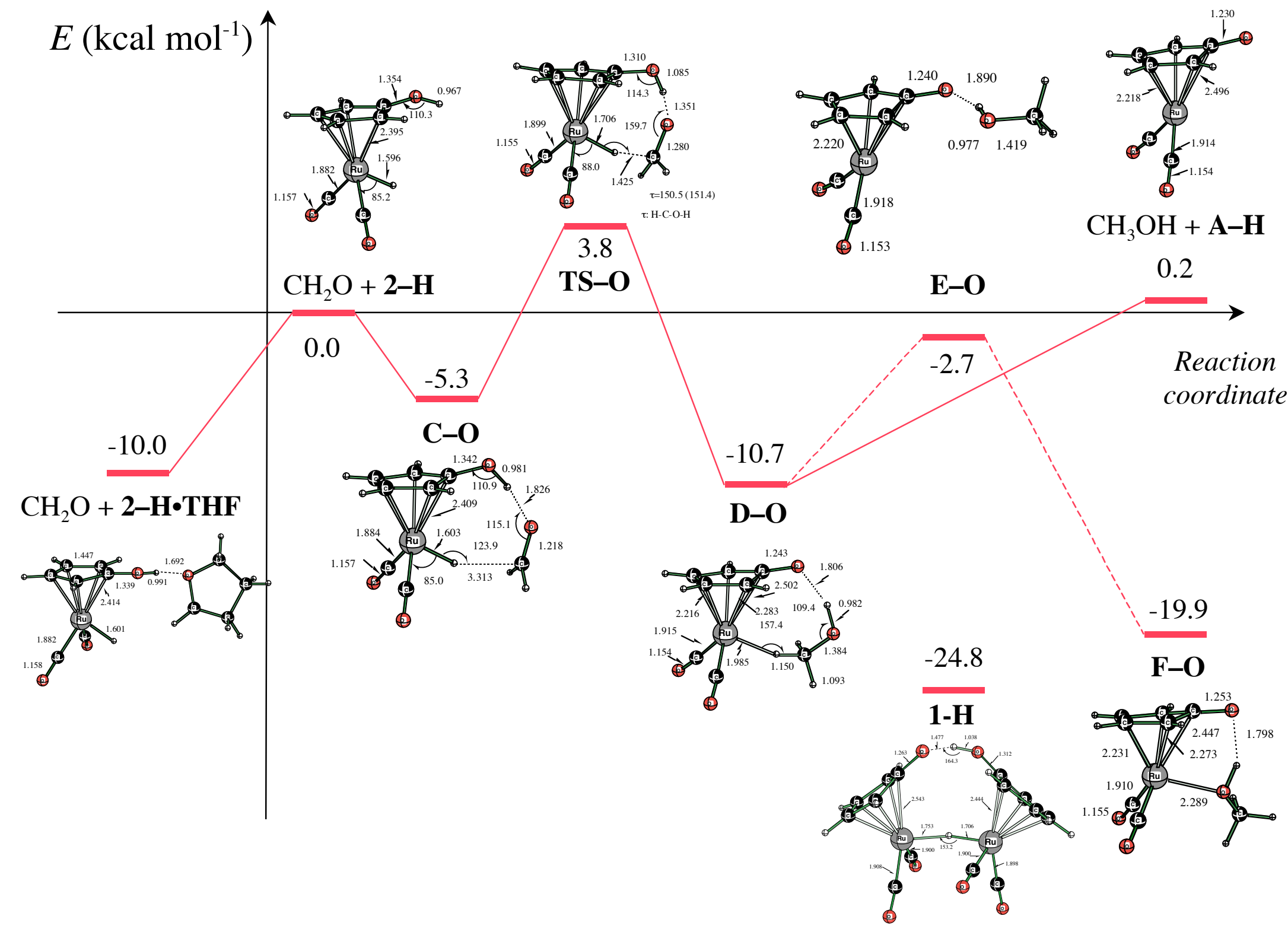


Figure S6. Schematic potential energy surface (based on the MP2 results shown in Table S1) for the reduction of formaldehyde by the model compound $\mathbf{2}-\mathbf{H}$. To be consistent during the entire process, the energy of a THF molecule infinitely apart from the calculated structure is included for all species after isolated reactant and $\mathbf{2}-\mathbf{H}$ (positive horizontal axis). The energy level shown for $\mathbf{1 - H}$ of $\mathbf{- 2 7 . 2}$ $\mathrm{kcal} \mathrm{mol}{ }^{-1}$ is actually for $\mathbf{1 - H}+\mathrm{NH}\left(\mathrm{CH}_{3}\right)_{2}$ vs. $\mathbf{2}-\mathbf{H}+\mathbf{2}-\mathbf{H}+\mathrm{H}_{2} \mathrm{C}=\mathrm{NCH}_{3}$.

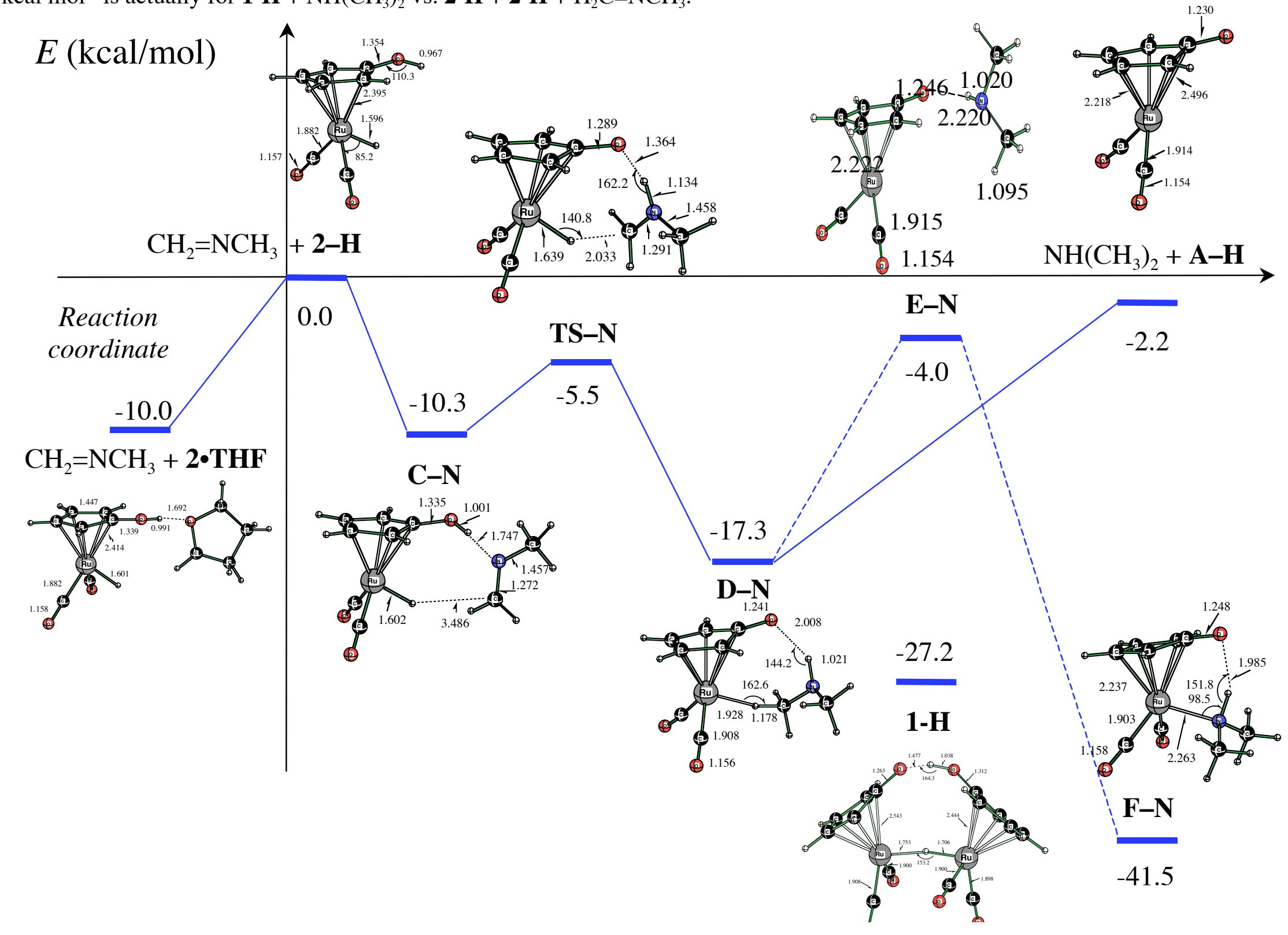


Table S3. xyz Coordinates for Species Optimized at B3LYP/BSII level

\section{2-H}

$\mathrm{Ru}-0.34278-0.00097-0.10222$

C $2.034360 .02153-0.38910$

C $1.628231 .13952 \quad 0.39057$

C $1.62521-1.172520 .27637$

C $1.07986-0.78203 \quad 1.55821$

C $1.08558 \quad 0.62825 \quad 1.62902$

O $2.717220 .14635-1.55136$

H $2.70864-0.68897-2.03761$

$\mathrm{H}-0.47997 \quad 0.02905-1.69237$

C $-1.67036-1.33251-0.17116$

C $-1.62837 \quad 1.37337-0.14274$

O - $-2.46627-2.17116-0.21885$

O -2.39791 $2.23640-0.17300$

H $1.81406-2.18417-0.05727$

H 1.815172 .173920 .13986

H $0.73166-1.461652 .32367$

H $0.74138 \quad 1.228132 .45987$

\section{$\mathrm{H}_{2} \mathrm{C}=\mathrm{O}$}

C $0.00000 \quad 0.00000-0.53175$

$\begin{array}{llllllll}\text { O } & 0.00000 & 0.00000 & 0.67794\end{array}$

H $0.00000 \quad 0.94137-1.11652$

H $0.00000-0.94137-1.11652$

\section{$\mathrm{CH}_{3} \mathrm{OH}$}

C $-0.04655 \quad 0.66698 \quad 0.00000$

O -0.04655 -0.758090 .00000$

H - $1.093520 .97743 \quad 0.00000$

H 0.440171 .079360 .89446

H $0.440171 .07936-0.89446$

H $0.86489-1.073310 .00000$ 


\begin{abstract}
A-H
Ru $0.304700 .00001-0.18862$

C - $2.184230 .00002-0.38036$

C $-1.58653-1.171640 .32094$

C - $1.58659 \quad 1.17151 \quad 0.32128$

C $-0.94250 \quad 0.72502 \quad 1.49564$

C - $0.94246-0.725451 .49543$

O - $2.859670 .00015-1.40865$

C $1.62171 \quad 1.38879-0.20861$

C $1.62182-1.38866-0.20869$

O $2.379822 .25592-0.13128$

O $2.38002-2.25572-0.13138$

H - 1.773232 .199960 .04226

H - $1.77310-2.200010 .04160$

H $-0.53467 \quad 1.34167 \quad 2.28626$

H -0.53458 - 1.342322 .28586
\end{abstract}

\title{
C-O
}

$\mathrm{Ru}-0.670080 .09840-0.02216$

C $1.18992-1.43168-0.05633$

C $0.99967-0.88174 \quad 1.25121$

C $-0.01278-2.09326-0.44042$

C $-0.89816-2.091980 .70221$

C $-0.28081-1.349831 .73411$

O $2.28130-1.37649-0.83452$

H $2.89994-0.68158-0.52237$

H $0.130601 .00857-1.07033$

C - $2.127470 .32098-1.19538$

C $-1.05303 \quad 1.708470 .87282$

O -3.01167 $0.43668-1.93260$

O -1.26127 2.698691 .43645

H - $0.16178-2.61291-1.37625$

H $1.74360-0.34657 \quad 1.82507$

H -1.86312 -2.57670 0.75277

H $-0.68603-1.162462 .71898$

O $3.801990 .87587-0.21143$

C $3.311301 .86751-0.71971$

H $3.809512 .84960-0.63623$

H $2.356371 .82337-1.27113$ 


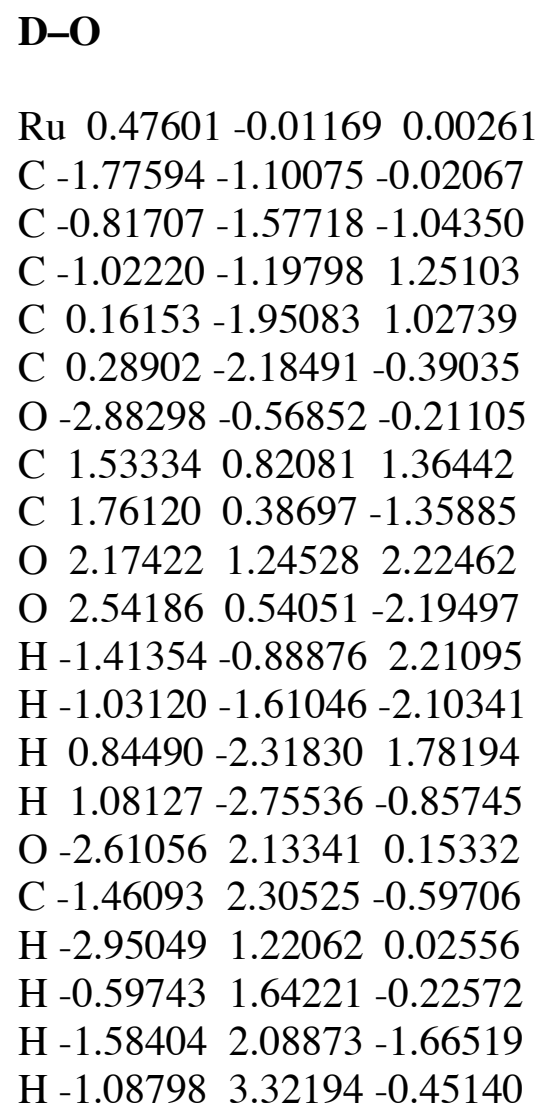

TS-O

Ru $0.46720-0.068750 .00458$ C - $1.78796-0.87722-0.04458$

C $-0.94998-1.60973-0.95879$

C - $1.19688-1.004871 .26906$

C $-0.10226-1.927821 .17235$

C $0.05183-2.30030-0.19540$

O -2.83183-0.16415-0.38600

H - $2.727950 .89338-0.16658$

H $-0.22008 \quad 1.46145-0.30362$

C $1.58203 \quad 0.76642 \quad 1.29565$

C $1.745520 .18516-1.37843$

O 2.244881 .246232 .11016

O $2.507670 .30076-2.23820$

H - $1.59268-0.562072 .17253$

H - $1.13265-1.69920-2.02062$

H $0.49390-2.293201 .99784$

H $0.77937-3.00091-0.58177$

$\begin{array}{llll}\mathrm{O}-2.32232 & 2.16245 & 0.05841\end{array}$

C - $1.21157 \quad 2.46631-0.49975$ 
H -1.13464 $2.43962-1.59884$

H -0.59388 $3.23571-0.01580$

\section{F-O}

$\mathrm{Ru}-0.27886-0.210790 .00638$

C $2.15713 \quad 0.01629-0.00816$

C $1.57996-0.28657 \quad 1.31580$

C $1.63538-1.05183-0.88428$

C $0.99307-2.04197-0.08321$

C $0.95708-1.569681 .27201$

O $2.717981 .07906-0.36464$

C - $1.56146-0.72374-1.31206$

C $-1.636200 .15551 \quad 1.29419$

O - $2.30088-1.10185-2.11476$

O - $2.42107 \quad 0.32267 \quad 2.12675$

H 1.84373 -1.12065 - 1.94358

H 1.744410 .314532 .20005

H $0.62052-3.00066-0.41968$

H $0.55518-2.119172 .11323$

O $0.17399 \quad 1.88614-0.79097$

C $-0.368693 .10049-0.25339$

H $0.006303 .95656-0.82371$

$\mathrm{H}-1.452813 .04520-0.36087$

H $-0.110193 .21857 \quad 0.80512$

H $1.165221 .90117-0.74789$

\section{E-O}

Ru $0.877470 .11618-0.15606$

C - $1.14629-1.302550 .09298$

C $0.14916-2.019810 .15376$

C - $1.01083-0.209771 .09099$

C $0.14686-0.446251 .86332$

C $0.87065-1.560041 .28128$

O -2.05707-1.46296-0.73237

C $1.24741 \quad 1.966860 .18471$

C $2.59056-0.18766-0.95561$

O $1.464393 .05810 \quad 0.48816$

O $3.64112-0.43932-1.36159$

$\mathrm{H}-1.77687 \quad 0.53487 \quad 1.26778$

H $0.40404-2.86246-0.47464$

H 0.452650 .093002 .75062

H $1.78554-1.979911 .67882$ 
H $-3.38122-0.14427-0.44987$

O $-3.82800 \quad 0.64266-0.08178$

C $-5.099660 .80361-0.69036$

H -5.56051 $1.69423-0.25366$

H $-5.022330 .95337-1.77725$

H -5.76065 -0.05489-0.50113

\section{2-H-THF}

$\mathrm{Ru}-1.524530 .18685-0.03261$

C $0.02855-1.65905-0.12098$

C $-0.19601-1.23308 \quad 1.22736$

C - $1.22956-2.02687-0.68408$

C - $2.21332-1.98757 \quad 0.37457$

C - $1.58241-1.501381 .54189$

O $1.18647-1.72617-0.78911$

H $1.90293-1.23235-0.31425$

H $-0.470651 .02215-0.90115$

C - $2.80040 \quad 0.82611-1.26011$

C $-1.66171 \quad 1.734501 .02634$

O $-3.57706 \quad 1.19970-2.03338$

O -1.71917 2.679951 .69386

H - 1.37326 -2.39795 -1.68893

H $0.57031-0.913341 .91979$

H -3.25016 -2.27974 0.28255

H -2.04709 -1.35448 2.50720

O $3.13277-0.284620 .35912$

C $3.02965 \quad 1.128390 .03934$

C $4.49105-0.748430 .14156$

C $4.26157 \quad 1.43873-0.80897$

C $5.314910 .50072-0.19441$

H $4.82611-1.262561 .04744$

H $4.47828-1.46521-0.68873$

H 3.029221 .697360 .97809

H $2.07613 \quad 1.28721-0.47057$

H 5.732610 .942440 .71738

H $4.545132 .49401-0.76271$

H $4.077931 .17907-1.85766$

H $6.143770 .27880-0.87242$

\section{1-H}

$\mathrm{Ru} 1.67603-0.370470 .08889$

C $1.966192 .00051-0.61069$

C 2.353811 .752890 .76994 
C $2.711111 .07706-1.44440$

C $3.699530 .42366-0.60909$

C 3.480960 .841090 .75333

O $1.060802 .86626-1.05276$

H $0.311823 .11635-0.31154$

H $0.02542-0.16298-0.33016$

C $1.71068-2.03263-0.82119$

C $1.32178-1.17127 \quad 1.76783$

O $1.77260-3.05303-1.40938$

O $1.16291-1.629552 .84335$

H $2.64340 \quad 1.03286-2.52134$

H 1.963092 .286311 .62406

H $4.48383-0.23671-0.95155$

H $4.073050 .54948 \quad 1.60954$

O $-0.62186 \quad 3.02061 \quad 0.69324$

C - $1.61915 \quad 2.21260 \quad 0.52009$

$\mathrm{Ru}-1.71055-0.25835-0.12310$

C $-2.22287 \quad 1.33125 \quad 1.53141$

C - $2.31104 \quad 1.89881-0.73958$

C -3.42065 0.752710 .97336

C - $3.476131 .09913-0.43047$

C $-1.77957-1.906230 .82275$

C - $1.79712-1.12209-1.80715$

O $-1.88623-2.91167 \quad 1.43187$

O - $1.89058-1.61421-2.87555$

H - $1.92872 \quad 1.310232 .57111$

H -2.09946 2.37515 - 1.68654

H -4.16430 $0.18058 \quad 1.51101$

H -4.27549 0.84431 - 1.11239

\section{$\mathrm{H}_{2} \mathrm{C}=\mathrm{NCH}_{3}$}

N $0.13921-0.53587-0.00007$

C $1.185260 .18234-0.00012$

H $1.16507 \quad 1.283890 .00017$

H $2.16403-0.299200 .00051$

C -1.15013 0.135220 .00006

$\mathrm{H}-1.71811-0.18751-0.87961$

H - $1.71740-0.186940 .88029$

H - $1.078851 .23552-0.00050$ 


\section{$\mathrm{NH}\left(\mathrm{CH}_{3}\right)_{2}$}

H $0.00000 \quad 1.343740 .50154$

$\begin{array}{llll}\mathrm{N} & 0.00000 & 0.56145 & -0.14636\end{array}$

C - $1.21849-0.222740 .02035$

$\mathrm{H}-1.28208-0.96854-0.78076$

H - $1.27808-0.762590 .98438$

H - $2.092380 .43062-0.06425$

C $1.21849-0.222740 .02035$

H $1.28208-0.96854-0.78076$

H $2.092380 .43062-0.06425$

H $1.27808-0.762590 .98438$

\section{C-N}

Ru $1.011300 .12476-0.03040$

C $-0.77999-1.49978-0.03154$

C $0.46627-2.11295-0.36122$

C $-0.63562-0.874851 .25068$

C $0.65518-1.258001 .77974$

C $1.32817-2.016230 .79527$

O - $1.85484-1.54020-0.82258$

H $0.176150 .94045-1.12780$

C $1.29543 \quad 1.789260 .79646$

C $2.471580 .37031-1.19450$

O $1.44150 \quad 2.81326 \quad 1.31938$

O $3.359180 .49963-1.92636$

H -1.41319 -0.34751 1.78519

H $0.65997-2.66685-1.26889$

H $1.03134-1.005752 .76173$

H $2.31386-2.450890 .88611$

$\mathrm{N}-3.634160 .49336-0.35838$

C -3.19447 $1.66076-0.60522$

$\mathrm{H}-2.135941 .79317-0.82925$

H -3.83975 $2.54897-0.60128$

C $-5.048480 .30791-0.06236$

H $-5.47513-0.38534-0.79468$

H $-5.14823-0.158740 .92321$

H $-5.616801 .24846-0.07826$

H -2.52562 - $0.83881-0.57729$ 


\section{TS-N}

Ru -0.74423 -0.10290 0.03154

C $0.982431 .69249-0.02842$

C $-0.02300 \quad 1.79639-1.07470$

C $0.23786 \quad 1.629391 .21672$

C - 1.141521 .939690 .94675

C - $1.300732 .04498-0.46145$

O $2.260321 .61078-0.17613$

H $2.848680 .40785-0.43589$

H $0.45210-1.19859-0.20427$

C - $1.35055-1.241221 .40945$

C - $1.73205-1.02868-1.28340$

O $-1.70583-1.922232 .27701$

O - $2.32942-1.57636-2.11244$

H 0.690941 .549062 .19568

H $0.198551 .87288-2.13110$

H -1.91773 2.08959 1.68509

H -2.21888 $2.28857-0.97913$

N $3.06795-0.70403-0.45893$

C $2.23650-1.40303-1.15753$

H $1.74973-0.94046-2.00718$

H $2.13452-2.47559-1.00706$

C $3.73060-1.248310 .72045$

H $4.76516-0.897490 .74763$

H $3.21720-0.886121 .61764$

H $3.71247-2.341520 .70875$

\section{D-N}

Ru -0.64700 -0.10143 0.01887

C $0.95470 \quad 1.84850 \quad 0.00490$

C $-0.22305 \quad 1.95319-0.89091$

C $0.36451 \quad 1.48931 \quad 1.31611$

C -1.043031 .693481 .26312$

C - $1.404671 .98397-0.09986$

O $2.155641 .88683-0.30619$

H $3.107440 .18299-0.78128$

H $0.91823-1.03304-0.61129$

C $-1.07331-1.434751 .31567$

C - $1.80454-0.88057-1.28480$

O $-1.35323-2.184802 .14933$

O $-2.54260-1.28374-2.07760$

H 0.945501 .301312 .20918

H -0.16041 2.17320 -1.94852 
$\begin{array}{lrrr}\text { H }-1.72987 & 1.67317 & 2.09917 \\ \text { H }-2.40521 & 2.21224 & -0.44363 \\ \text { N } & 3.06673 & -0.83516 & -0.71302 \\ \text { C } & 1.86296 & -1.34251 & -1.24329 \\ \text { H } & 1.67607 & -0.97775 & -2.25356 \\ \text { H } & 1.82617 & -2.43522 & -1.21405 \\ \text { C } & 3.40572 & -1.29146 & 0.62632 \\ \text { H } & 4.34526 & -0.83115 & 0.94104 \\ \text { H } & 2.62767 & -1.03830 & 1.36889 \\ \text { H } & 3.53603 & -2.37932 & 0.62711\end{array}$

F-N

Ru $0.11699-0.409950 .00135$

C -2.12594 0.68404-0.00726

C - $1.82995-0.18978-1.16267$

C $-1.83100-0.173411 .16086$

C - $1.68069-1.528190 .72374$

C - $1.67981-1.53834-0.70626$

O $-2.326191 .91595-0.01616$

C $1.31369-0.964811 .37237$

C $1.31228-0.97948-1.36470$

O $1.99091-1.340652 .23292$

O $1.98788-1.36430-2.22255$

H -1.92274 0.153182 .18859

H - $1.920940 .12216-2.19501$

H -1.61102 -2.40406 1.35556

H - $1.61008-2.42320-1.32540$

N $0.605461 .80015-0.00883$

H -0.35776 2.16837 -0.02260

C $1.257842 .31434 \quad 1.21621$

C $1.289352 .29984-1.22257$

H 1.324963 .410581 .19562

H 0.679812 .008432 .08924

H 2.266731 .901731 .29584

H $1.361203 .39592-1.21073$

H $2.297891 .88202-1.27440$

H $0.730811 .98836-2.10632$

\section{E-N}

$\mathrm{Ru} 1.170480 .13020-0.13351$

C $-0.74313-1.45939-0.15962$

C $0.60390-2.07563-0.04216$

C $-0.77331-0.439450 .92475$ 
$\begin{array}{lccc}\text { C } & 0.33515 & -0.64911 & 1.77218 \\ \text { C } & 1.19306 & -1.65374 & 1.17217 \\ \text { O } & -1.57225 & -1.62432 & -1.06116 \\ \text { C } & 1.35120 & 1.97032 & 0.36497 \\ \text { C } & 2.95735 & 0.03228 & -0.81246 \\ \text { O } & 1.44770 & 3.04953 & 0.76250 \\ \text { O } & 4.05399 & -0.09679 & -1.14948 \\ \text { H }-1.61815 & 0.22095 & 1.08719 \\ \text { H } & 0.97904 & -2.84178 & -0.70734 \\ \text { H } & 0.52664 & -0.16128 & 2.71940 \\ \text { H } & 2.10682 & -2.03044 & 1.61342 \\ \text { H }-3.31049 & -0.30902 & -0.63637 \\ \text { N }-3.85620 & 0.38101 & -0.12057 \\ \text { C }-5.04359 & -0.25296 & 0.44317 \\ \text { H }-5.54855 & 0.44484 & 1.12250 \\ \text { H }-5.78119 & -0.57094 & -0.31735 \\ \text { H }-4.75122 & -1.13393 & 1.02340 \\ \text { C }-4.17668 & 1.50881 & -0.98933 \\ \text { H }-4.63444 & 2.31220 & -0.39909 \\ \text { H }-3.25874 & 1.90353 & -1.43658 \\ \text { H }-4.87679 & 1.25595 & -1.80755\end{array}$ 
X-ray Crystal Structure Determination of $\left[2,5-\mathrm{Ph}_{2}-3,4-\mathrm{Tol}_{2}\left(\eta^{4}-\right.\right.$ $\left.\left.\mathrm{C}_{4} \mathrm{CO}\right)\right](\mathrm{CO})_{2} \mathrm{RuNH}_{2} \mathrm{C}_{6} \mathrm{H}_{4}-p-\mathrm{NHCH}_{2} \mathrm{Ph}(9)$. X-ray quality crystals were grown by cooling a $\mathrm{CH}_{2} \mathrm{Cl}_{2} /$ hexane solution of 9 to $-20{ }^{\circ} \mathrm{C}$ for one week. A colorless crystal with approximate dimensions $0.20 \times 0.02 \times 0.02 \mathrm{~mm}^{3}$ was selected under oil under ambient conditions and attached to the tip of a nylon loop. The crystal was mounted in a stream of cold nitrogen at 100(2) K and centered in the X-ray beam by using a video camera.

The crystal evaluation and data collection were performed on a Bruker CCD-1000 diffractometer with $\operatorname{Mo~}_{\alpha}(\lambda=0.71073 \AA)$ radiation and the diffractometer to crystal distance of $4.9 \mathrm{~cm}$.

The initial cell constants were obtained from three series of $\omega$ scans at different starting angles. Each series consisted of 20 frames collected at intervals of $0.3^{\circ}$ in a $6^{\circ}$ range about $\omega$ with the exposure time of 10 seconds per frame. A total of 67 reflections were obtained. The reflections were successfully indexed by an automated indexing routine built into the SMART program. The final cell constants were calculated from a set of 3249 strong reflections from the actual data collection.

The data were collected by using the hemisphere data collection routine. The reciprocal space was surveyed to the extent of a full sphere to a resolution of $0.80 \AA$. A total of 14798 data were harvested by collecting three sets of frames with $0.30^{\circ}$ scans in $\omega$ with an exposure time 90 sec per frame. These highly redundant datasets were corrected for Lorentz and polarization effects. The absorption correction was based on fitting a function to the empirical transmission surface as sampled by multiple equivalent measurements. ${ }^{11}$ 
The systematic absences in the diffraction data were consistent with the space groups $P 2_{1}$ and $P 2_{1} / \mathrm{m}$. The E-statistics strongly suggested the non-centrosymmetric space group $P 2_{1}$ that yielded chemically reasonable and computationally stable results of refinement.

A successful solution by the direct methods provided most non-hydrogen atoms from the $E$-map. The remaining non-hydrogen atoms were located in an alternating series of least-squares cycles and difference Fourier maps. All non-hydrogen atoms were refined with anisotropic displacement coefficients. All hydrogen atoms were included in the structure factor calculation at idealized positions and were allowed to ride on the neighboring atoms with relative isotropic displacement coefficients.

The final least-squares refinement of 471 parameters against 5737 data resulted in residuals $R$ (based on $F^{2}$ for $I \geq 2 \sigma$ ) and $w R$ (based on $F^{2}$ for all data) of 0.0466 and 0.0924 , respectively. The final difference Fourier map was featureless. 
Figure S7. Molecular drawings of 9 shown with $30 \%$ probability ellipsoids.
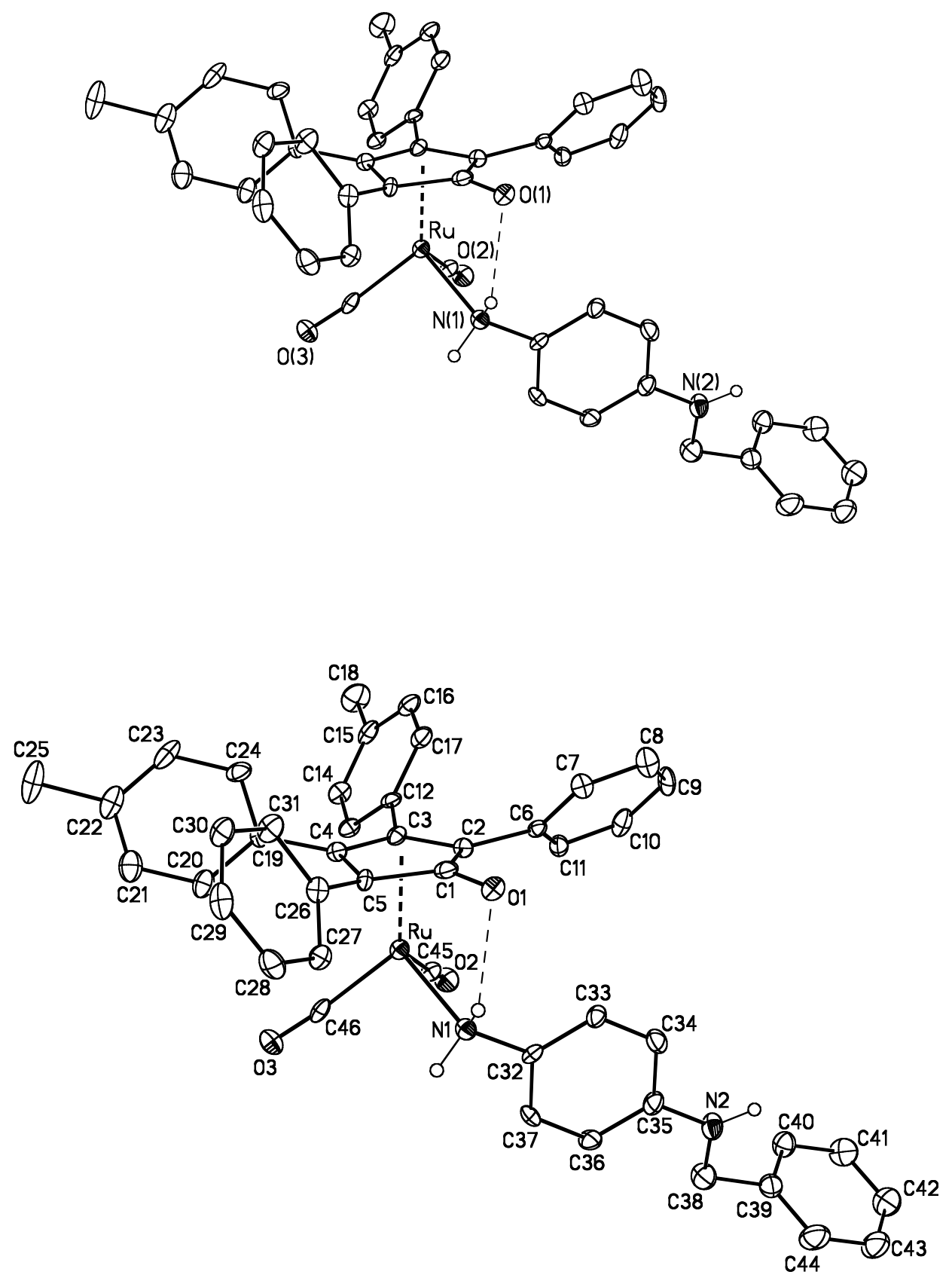
Table S4. Crystal data and structure refinement for $\left[2,5-\mathrm{Ph}_{2}-3,4-\mathrm{Tol}_{2}\left(\eta^{4}-\right.\right.$ $\left.\left.\mathrm{C}_{4} \mathrm{CO}\right)\right](\mathrm{CO})_{2} \mathrm{RuNH}_{2} \mathrm{C}_{6} \mathrm{H}_{4}-p-\mathrm{NHCH}_{2} \mathrm{Ph}(\mathbf{9})$.

\begin{tabular}{|c|c|c|}
\hline Empirical formula & \multicolumn{2}{|l|}{$\mathrm{C}_{46} \mathrm{H}_{38} \mathrm{~N}_{2} \mathrm{O}_{3} \mathrm{Ru}$} \\
\hline Formula weight & \multicolumn{2}{|l|}{767.85} \\
\hline Temperature & \multicolumn{2}{|l|}{$100(2) \mathrm{K}$} \\
\hline Wavelength & \multicolumn{2}{|l|}{$0.71073 \AA$} \\
\hline Crystal system & \multicolumn{2}{|l|}{ Monoclinic } \\
\hline Space group & \multicolumn{2}{|l|}{$\mathrm{P} 2_{1}$} \\
\hline \multirow[t]{3}{*}{ Unit cell dimensions } & $\mathrm{a}=11.719(3) \AA$ & $\alpha=90^{\circ}$. \\
\hline & $\mathrm{b}=9.906(2) \AA$ & $\beta=97.938(10)^{\circ}$. \\
\hline & $\mathrm{c}=17.403(5) \AA$ & $\gamma=90^{\circ}$. \\
\hline Volume & \multicolumn{2}{|l|}{$2000.9(10) \AA^{3}$} \\
\hline $\mathrm{Z}$ & \multicolumn{2}{|l|}{2} \\
\hline Density (calculated) & \multicolumn{2}{|l|}{$1.274 \mathrm{Mg} / \mathrm{m}^{3}$} \\
\hline Absorption coefficient & \multicolumn{2}{|l|}{$0.433 \mathrm{~mm}^{-1}$} \\
\hline $\mathrm{F}(000)$ & \multicolumn{2}{|l|}{792} \\
\hline Crystal size & \multicolumn{2}{|c|}{$0.40 \times 0.31 \times 0.17 \mathrm{~mm}^{3}$} \\
\hline Theta range for data collection & \multicolumn{2}{|c|}{2.25 to $24.88^{\circ}$. } \\
\hline Index ranges & \multicolumn{2}{|c|}{$-13<=\mathrm{h}<=13,-11<=\mathrm{k}<=11,-20<=\mathrm{l}<=20$} \\
\hline Reflections collected & \multicolumn{2}{|l|}{14798} \\
\hline Independent reflections & \multicolumn{2}{|c|}{$5737[\mathrm{R}(\mathrm{int})=0.0696]$} \\
\hline Completeness to theta $=24.88^{\circ}$ & \multicolumn{2}{|l|}{$99.3 \%$} \\
\hline Absorption correction & \multicolumn{2}{|c|}{ Multi-scan with SADABS } \\
\hline Max. and min. transmission & \multicolumn{2}{|c|}{0.9301 and 0.8459} \\
\hline Refinement method & \multicolumn{2}{|c|}{ Full-matrix least-squares on $\mathrm{F}^{2}$} \\
\hline Data / restraints / parameters & \multicolumn{2}{|c|}{$5737 / 1 / 471$} \\
\hline Goodness-of-fit on $\mathrm{F}^{2}$ & \multicolumn{2}{|l|}{0.964} \\
\hline Final $R$ indices $[I>2 \operatorname{sigma}(I)]$ & \multicolumn{2}{|c|}{$\mathrm{R} 1=0.0466, \mathrm{wR} 2=0.0833$} \\
\hline $\mathrm{R}$ indices (all data) & \multicolumn{2}{|c|}{$\mathrm{R} 1=0.0779, \mathrm{wR} 2=0.0924$} \\
\hline
\end{tabular}


Absolute structure parameter

Largest diff. peak and hole
$0.01(4)$

0.810 and -0.568 e. $\AA^{-3}$

Table S5. Atomic coordinates $\left(\mathrm{x} 10^{4}\right)$ and equivalent isotropic displacement parameters $\left(\AA^{2} \mathrm{x}\right.$ $10^{3}$ ) for $\left[2,5-\mathrm{Ph}_{2}-3,4-\mathrm{Tol}_{2}\left(\eta^{4}-\mathrm{C}_{4} \mathrm{CO}\right)\right](\mathrm{CO})_{2} \mathrm{RuNH}_{2} \mathrm{C}_{6} \mathrm{H}_{4}-p-\mathrm{NHCH}_{2} \mathrm{Ph}(\mathbf{9})$. U(eq) is defined as one third of the trace of the orthogonalized $\mathrm{U}^{\mathrm{ij}}$ tensor.

\begin{tabular}{|c|c|c|c|c|}
\hline & $\mathrm{X}$ & $\mathrm{y}$ & $\mathrm{z}$ & $\mathrm{U}(\mathrm{eq})$ \\
\hline $\mathrm{Ru}$ & $8790(1)$ & $8004(1)$ & $1025(1)$ & $21(1)$ \\
\hline $\mathrm{O}(1)$ & $9936(3)$ & $10927(4)$ & $639(2)$ & $24(1)$ \\
\hline $\mathrm{O}(2)$ & $6331(4)$ & $6990(4)$ & $737(2)$ & $37(1)$ \\
\hline $\mathrm{O}(3)$ & $9550(4)$ & $5088(4)$ & $1152(3)$ & $33(1)$ \\
\hline $\mathrm{N}(1)$ & $9068(4)$ & $8374(4)$ & $-165(2)$ & $24(1)$ \\
\hline $\mathrm{N}(2)$ & $5295(5)$ & $9315(5)$ & $-2477(3)$ & $39(1)$ \\
\hline$C(1)$ & $9636(7)$ & $10219(8)$ & $1182(4)$ & $26(2)$ \\
\hline$C(2)$ & $8480(5)$ & $10126(6)$ & 1411(3) & $20(1)$ \\
\hline $\mathrm{C}(3)$ & $8512(5)$ & $9158(5)$ & $2037(3)$ & $21(1)$ \\
\hline $\mathrm{C}(4)$ & $9644(5)$ & $8602(5)$ & $2178(3)$ & $20(1)$ \\
\hline $\mathrm{C}(5)$ & $10306(5)$ & $9149(5)$ & $1608(3)$ & $22(1)$ \\
\hline $\mathrm{C}(6)$ & $7523(5)$ & $11088(5)$ & 1139(3) & $20(1)$ \\
\hline $\mathrm{C}(7)$ & $7781(6)$ & $12441(6)$ & $1102(3)$ & $28(2)$ \\
\hline $\mathrm{C}(8)$ & $6913(6)$ & $13376(6)$ & 919(4) & $40(2)$ \\
\hline $\mathrm{C}(9)$ & $5775(5)$ & $12984(10)$ & $767(3)$ & $34(1)$ \\
\hline$C(10)$ & $5507(6)$ & $11624(7)$ & $800(3)$ & $36(2)$ \\
\hline $\mathrm{C}(11)$ & $6387(6)$ & $10687(6)$ & $987(3)$ & $26(2)$ \\
\hline$C(12)$ & $7606(5)$ & $8985(6)$ & $2550(3)$ & $23(2)$ \\
\hline$C(13)$ & $7262(5)$ & $7688(6)$ & $2736(3)$ & $26(2)$ \\
\hline $\mathrm{C}(14)$ & $6512(6)$ & $7516(6)$ & $3278(3)$ & $31(2)$ \\
\hline$C(15)$ & $6097(6)$ & $8596(7)$ & $3648(4)$ & $33(2)$ \\
\hline$C(16)$ & $6427(5)$ & $9863(7)$ & $3454(3)$ & $32(2)$ \\
\hline $\mathrm{C}(17)$ & $7189(5)$ & $10067(7)$ & $2910(3)$ & $31(2)$ \\
\hline $\mathrm{C}(18)$ & $5307(6)$ & 8386(7) & $4253(4)$ & $54(3)$ \\
\hline C(19) & $10128(5)$ & $7736(6)$ & 2841(3) & $20(2)$ \\
\hline$C(20)$ & $10895(6)$ & $6706(6)$ & $2746(4)$ & $33(2)$ \\
\hline $\mathrm{C}(21)$ & $11416(6)$ & $5977(7)$ & $3372(4)$ & $40(2)$ \\
\hline $\mathrm{C}(22)$ & $11202(7)$ & $6238(7)$ & $4105(4)$ & $44(2)$ \\
\hline $\mathrm{C}(23)$ & $10457(7)$ & 7293(7) & $4213(4)$ & $45(2)$ \\
\hline $\mathrm{C}(24)$ & 9914(4) & $8014(10)$ & $3585(3)$ & $33(1)$ \\
\hline$C(25)$ & $11793(7)$ & $5467(8)$ & $4802(4)$ & $70(3)$ \\
\hline
\end{tabular}




$\begin{array}{lrrrr}\mathrm{C}(26) & 11577(5) & 9042(6) & 1660(3) & 26(2) \\ \mathrm{C}(27) & 12116(5) & 8687(5) & 1033(3) & 27(2) \\ \mathrm{C}(28) & 13329(6) & 8658(6) & 1117(4) & 34(2) \\ \mathrm{C}(29) & 13969(6) & 8958(6) & 1814(4) & 34(2) \\ \mathrm{C}(30) & 13433(5) & 9342(6) & 2427(4) & 32(2) \\ \mathrm{C}(31) & 12253(5) & 9386(6) & 2350(4) & 32(2) \\ \mathrm{C}(32) & 8101(5) & 8643(6) & -754(3) & 22(2) \\ \mathrm{C}(33) & 7632(6) & 9900(6) & -866(3) & 28(2) \\ \mathrm{C}(34) & 6721(6) & 10135(6) & -1435(4) & 33(2) \\ \mathrm{C}(35) & 6225(6) & 9076(7) & -1901(4) & 32(2) \\ \mathrm{C}(36) & 6710(5) & 7803(8) & -1785(3) & 31(2) \\ \mathrm{C}(37) & 7630(6) & 7592(5) & -1213(4) & 26(2) \\ \mathrm{C}(38) & 4637(6) & 8200(9) & -2831(4) & 44(2) \\ \mathrm{C}(39) & 3586(6) & 8724(6) & -3377(4) & 39(2) \\ \mathrm{C}(40) & 2587(6) & 8999(6) & -3086(4) & 41(2) \\ \mathrm{C}(41) & 1626(7) & 9510(7) & -3585(5) & 51(2) \\ \mathrm{C}(42) & 1727(7) & 9682(7) & -4353(5) & 55(2) \\ \mathrm{C}(43) & 2734(8) & 9397(8) & -4643(5) & 63(2) \\ \mathrm{C}(44) & 3650(7) & 8877(8) & -4142(4) & 57(2) \\ \mathrm{C}(45) & 7275(6) & 7374(6) & 808(3) & 21(2) \\ \mathrm{C}(46) & 9298(7) & 6188(7) & 1066(4) & 26(2)\end{array}$


Table S6. Bond lengths $[\AA]$ and angles $\left[^{\circ}\right]$ for $\left[2,5-\mathrm{Ph}_{2}-3,4-\mathrm{Tol}_{2}\left(\eta^{4}-\mathrm{C}_{4} \mathrm{CO}\right)\right](\mathrm{CO})_{2} \mathrm{RuNH}_{2} \mathrm{C}_{6} \mathrm{H}_{4}-p-$ $\mathrm{NHCH}_{2} \mathrm{Ph}(\mathbf{9})$.

\begin{tabular}{|c|c|c|c|}
\hline $\mathrm{Ru}-\mathrm{C}(45)$ & $1.871(7)$ & $\mathrm{C}(13)-\mathrm{C}(14)$ & $1.387(8)$ \\
\hline Ru-C(46) & $1.893(7)$ & $\mathrm{C}(14)-\mathrm{C}(15)$ & $1.372(8)$ \\
\hline $\mathrm{Ru}-\mathrm{C}(3)$ & $2.161(5)$ & $C(15)-C(16)$ & $1.369(8)$ \\
\hline $\mathrm{Ru}-\mathrm{N}(1)$ & $2.172(4)$ & $\mathrm{C}(15)-\mathrm{C}(18)$ & $1.510(8)$ \\
\hline $\mathrm{Ru}-\mathrm{C}(4)$ & $2.196(6)$ & $C(16)-C(17)$ & $1.404(8)$ \\
\hline $\mathrm{Ru}-\mathrm{C}(5)$ & $2.230(6)$ & $\mathrm{C}(19)-\mathrm{C}(24)$ & $1.380(7)$ \\
\hline $\mathrm{Ru}-\mathrm{C}(2)$ & $2.250(5)$ & $C(19)-C(20)$ & $1.385(8)$ \\
\hline $\mathrm{Ru}-\mathrm{C}(1)$ & $2.407(8)$ & $\mathrm{C}(20)-\mathrm{C}(21)$ & $1.378(8)$ \\
\hline $\mathrm{O}(1)-\mathrm{C}(1)$ & $1.264(8)$ & $\mathrm{C}(21)-\mathrm{C}(22)$ & $1.359(9)$ \\
\hline $\mathrm{O}(2)-\mathrm{C}(45)$ & $1.160(6)$ & $C(22)-C(23)$ & $1.391(10)$ \\
\hline $\mathrm{O}(3)-\mathrm{C}(46)$ & $1.134(7)$ & $\mathrm{C}(22)-\mathrm{C}(25)$ & $1.518(9)$ \\
\hline $\mathrm{N}(1)-\mathrm{C}(32)$ & $1.444(7)$ & $\mathrm{C}(23)-\mathrm{C}(24)$ & $1.384(9)$ \\
\hline $\mathrm{N}(2)-\mathrm{C}(35)$ & $1.395(8)$ & $C(26)-C(27)$ & $1.379(8)$ \\
\hline $\mathrm{N}(2)-\mathrm{C}(38)$ & $1.436(9)$ & $\mathrm{C}(26)-\mathrm{C}(31)$ & $1.386(8)$ \\
\hline $\mathrm{C}(1)-\mathrm{C}(5)$ & $1.459(9)$ & $\mathrm{C}(27)-\mathrm{C}(28)$ & $1.409(8)$ \\
\hline$C(1)-C(2)$ & $1.467(9)$ & $\mathrm{C}(28)-\mathrm{C}(29)$ & $1.368(8)$ \\
\hline$C(2)-C(3)$ & $1.449(8)$ & $\mathrm{C}(29)-\mathrm{C}(30)$ & $1.365(8)$ \\
\hline$C(2)-C(6)$ & $1.499(8)$ & $\mathrm{C}(30)-\mathrm{C}(31)$ & $1.372(8)$ \\
\hline$C(3)-C(4)$ & $1.426(8)$ & $\mathrm{C}(32)-\mathrm{C}(33)$ & $1.364(8)$ \\
\hline $\mathrm{C}(3)-\mathrm{C}(12)$ & $1.488(8)$ & $\mathrm{C}(32)-\mathrm{C}(37)$ & $1.381(7)$ \\
\hline$C(4)-C(5)$ & $1.447(7)$ & $\mathrm{C}(33)-\mathrm{C}(34)$ & $1.372(9)$ \\
\hline C(4)-C(19) & $1.486(7)$ & $\mathrm{C}(34)-\mathrm{C}(35)$ & $1.403(8)$ \\
\hline$C(5)-C(26)$ & $1.483(8)$ & $C(35)-C(36)$ & $1.386(9)$ \\
\hline$C(6)-C(7)$ & $1.378(7)$ & $\mathrm{C}(36)-\mathrm{C}(37)$ & $1.379(8)$ \\
\hline$C(6)-C(11)$ & $1.379(8)$ & $\mathrm{C}(38)-\mathrm{C}(39)$ & $1.538(9)$ \\
\hline$C(7)-C(8)$ & $1.380(8)$ & $\mathrm{C}(39)-\mathrm{C}(44)$ & $1.352(9)$ \\
\hline C(8)-C(9) & $1.379(8)$ & $\mathrm{C}(39)-\mathrm{C}(40)$ & $1.366(9)$ \\
\hline C(9)-C(10) & $1.386(11)$ & $\mathrm{C}(40)-\mathrm{C}(41)$ & $1.417(9)$ \\
\hline $\mathrm{C}(10)-\mathrm{C}(11)$ & $1.392(8)$ & $\mathrm{C}(41)-\mathrm{C}(42)$ & $1.369(9)$ \\
\hline $\mathrm{C}(12)-\mathrm{C}(17)$ & $1.366(8)$ & $\mathrm{C}(42)-\mathrm{C}(43)$ & $1.375(10)$ \\
\hline $\mathrm{C}(12)-\mathrm{C}(13)$ & $1.398(8)$ & $\mathrm{C}(43)-\mathrm{C}(44)$ & $1.385(10)$ \\
\hline $\mathrm{C}(45)-\mathrm{Ru}-\mathrm{C}(46)$ & $88.6(3)$ & $\mathrm{N}(1)-\mathrm{Ru}-\mathrm{C}(4)$ & $135.76(19)$ \\
\hline $\mathrm{C}(45)-\mathrm{Ru}-\mathrm{C}(3)$ & $95.5(2)$ & $\mathrm{C}(45)-\mathrm{Ru}-\mathrm{C}(5)$ & $159.4(2)$ \\
\hline C(46)-Ru-C(3) & $123.6(2)$ & $\mathrm{C}(46)-\mathrm{Ru}-\mathrm{C}(5)$ & $103.8(3)$ \\
\hline $\mathrm{C}(45)-\mathrm{Ru}-\mathrm{N}(1)$ & $97.3(2)$ & $\mathrm{C}(3)-\mathrm{Ru}-\mathrm{C}(5)$ & $64.0(2)$ \\
\hline $\mathrm{C}(46)-\mathrm{Ru}-\mathrm{N}(1)$ & $96.2(2)$ & $\mathrm{N}(1)-\mathrm{Ru}-\mathrm{C}(5)$ & $97.64(18)$ \\
\hline C(3)-Ru-N(1) & $138.37(18)$ & C(4)-Ru-C(5) & 38.14(19) \\
\hline $\mathrm{C}(45)-\mathrm{Ru}-\mathrm{C}(4)$ & $125.1(2)$ & $\mathrm{C}(45)-\mathrm{Ru}-\mathrm{C}(2)$ & $100.6(2)$ \\
\hline $\mathrm{C}(46)-\mathrm{Ru}-\mathrm{C}(4)$ & $97.0(3)$ & $\mathrm{C}(46)-\mathrm{Ru}-\mathrm{C}(2)$ & $159.9(3)$ \\
\hline $\mathrm{C}(3)-\mathrm{Ru}-\mathrm{C}(4)$ & $38.18(19)$ & $\mathrm{C}(3)-\mathrm{Ru}-\mathrm{C}(2)$ & $38.3(2)$ \\
\hline
\end{tabular}




\begin{tabular}{|c|c|c|c|}
\hline $\mathrm{N}(1)-\mathrm{Ru}-\mathrm{C}(2)$ & $100.29(18)$ & $\mathrm{C}(9)-\mathrm{C}(8)-\mathrm{C}(7)$ & $121.2(6)$ \\
\hline $\mathrm{C}(4)-\mathrm{Ru}-\mathrm{C}(2)$ & $63.1(2)$ & $\mathrm{C}(8)-\mathrm{C}(9)-\mathrm{C}(10)$ & $118.9(7)$ \\
\hline $\mathrm{C}(5)-\mathrm{Ru}-\mathrm{C}(2)$ & $62.7(2)$ & $C(9)-C(10)-C(11)$ & $119.6(6)$ \\
\hline $\mathrm{C}(45)-\mathrm{Ru}-\mathrm{C}(1)$ & $133.8(3)$ & $\mathrm{C}(6)-\mathrm{C}(11)-\mathrm{C}(10)$ & $121.1(6)$ \\
\hline $\mathrm{C}(46)-\mathrm{Ru}-\mathrm{C}(1)$ & $137.7(3)$ & $C(17)-C(12)-C(13)$ & $118.9(5)$ \\
\hline $\mathrm{C}(3)-\mathrm{Ru}-\mathrm{C}(1)$ & $62.1(2)$ & $\mathrm{C}(17)-\mathrm{C}(12)-\mathrm{C}(3)$ & $121.0(6)$ \\
\hline $\mathrm{N}(1)-\mathrm{Ru}-\mathrm{C}(1)$ & $80.8(2)$ & $\mathrm{C}(13)-\mathrm{C}(12)-\mathrm{C}(3)$ & $119.8(5)$ \\
\hline $\mathrm{C}(4)-\mathrm{Ru}-\mathrm{C}(1)$ & $61.5(2)$ & $\mathrm{C}(14)-\mathrm{C}(13)-\mathrm{C}(12)$ & $120.0(5)$ \\
\hline $\mathrm{C}(5)-\mathrm{Ru}-\mathrm{C}(1)$ & $36.4(2)$ & $C(15)-C(14)-C(13)$ & $121.6(5)$ \\
\hline $\mathrm{C}(2)-\mathrm{Ru}-\mathrm{C}(1)$ & $36.5(2)$ & $C(16)-C(15)-C(14)$ & $117.9(5)$ \\
\hline $\mathrm{C}(32)-\mathrm{N}(1)-\mathrm{Ru}$ & $120.1(3)$ & $\mathrm{C}(16)-\mathrm{C}(15)-\mathrm{C}(18)$ & $121.3(6)$ \\
\hline $\mathrm{C}(35)-\mathrm{N}(2)-\mathrm{C}(38)$ & $119.8(5)$ & $\mathrm{C}(14)-\mathrm{C}(15)-\mathrm{C}(18)$ & $120.7(6)$ \\
\hline $\mathrm{O}(1)-\mathrm{C}(1)-\mathrm{C}(5)$ & $126.7(6)$ & $C(15)-C(16)-C(17)$ & $121.8(6)$ \\
\hline $\mathrm{O}(1)-\mathrm{C}(1)-\mathrm{C}(2)$ & $126.9(7)$ & $\mathrm{C}(12)-\mathrm{C}(17)-\mathrm{C}(16)$ & $119.8(6)$ \\
\hline $\mathrm{C}(5)-\mathrm{C}(1)-\mathrm{C}(2)$ & $105.5(6)$ & $\mathrm{C}(24)-\mathrm{C}(19)-\mathrm{C}(20)$ & $117.7(6)$ \\
\hline $\mathrm{O}(1)-\mathrm{C}(1)-\mathrm{Ru}$ & $125.0(5)$ & $\mathrm{C}(24)-\mathrm{C}(19)-\mathrm{C}(4)$ & $120.9(6)$ \\
\hline$C(5)-C(1)-R u$ & $65.1(4)$ & $\mathrm{C}(20)-\mathrm{C}(19)-\mathrm{C}(4)$ & $121.1(5)$ \\
\hline $\mathrm{C}(2)-\mathrm{C}(1)-\mathrm{Ru}$ & $65.9(3)$ & $\mathrm{C}(21)-\mathrm{C}(20)-\mathrm{C}(19)$ & $121.0(6)$ \\
\hline$C(3)-C(2)-C(1)$ & $108.5(5)$ & $\mathrm{C}(22)-\mathrm{C}(21)-\mathrm{C}(20)$ & $121.6(7)$ \\
\hline$C(3)-C(2)-C(6)$ & $126.8(5)$ & $\mathrm{C}(21)-\mathrm{C}(22)-\mathrm{C}(23)$ & $118.1(6)$ \\
\hline$C(1)-C(2)-C(6)$ & $123.6(5)$ & $\mathrm{C}(21)-\mathrm{C}(22)-\mathrm{C}(25)$ & $122.1(7)$ \\
\hline$C(3)-C(2)-R u$ & $67.5(3)$ & $\mathrm{C}(23)-\mathrm{C}(22)-\mathrm{C}(25)$ & $119.7(7)$ \\
\hline$C(1)-C(2)-R u$ & $77.6(4)$ & $\mathrm{C}(24)-\mathrm{C}(23)-\mathrm{C}(22)$ & $120.6(7)$ \\
\hline$C(6)-C(2)-R u$ & $130.1(4)$ & $\mathrm{C}(19)-\mathrm{C}(24)-\mathrm{C}(23)$ & $121.0(7)$ \\
\hline $\mathrm{C}(4)-\mathrm{C}(3)-\mathrm{C}(2)$ & $108.1(5)$ & $\mathrm{C}(27)-\mathrm{C}(26)-\mathrm{C}(31)$ & $118.5(6)$ \\
\hline $\mathrm{C}(4)-\mathrm{C}(3)-\mathrm{C}(12)$ & $125.2(5)$ & $\mathrm{C}(27)-\mathrm{C}(26)-\mathrm{C}(5)$ & $122.4(6)$ \\
\hline $\mathrm{C}(2)-\mathrm{C}(3)-\mathrm{C}(12)$ & $125.6(5)$ & $\mathrm{C}(31)-\mathrm{C}(26)-\mathrm{C}(5)$ & $118.9(5)$ \\
\hline $\mathrm{C}(4)-\mathrm{C}(3)-\mathrm{Ru}$ & $72.2(3)$ & $\mathrm{C}(26)-\mathrm{C}(27)-\mathrm{C}(28)$ & $119.4(6)$ \\
\hline$C(2)-C(3)-R u$ & $74.2(3)$ & $\mathrm{C}(29)-\mathrm{C}(28)-\mathrm{C}(27)$ & $120.5(6)$ \\
\hline $\mathrm{C}(12)-\mathrm{C}(3)-\mathrm{Ru}$ & $128.9(4)$ & $\mathrm{C}(30)-\mathrm{C}(29)-\mathrm{C}(28)$ & $119.9(6)$ \\
\hline $\mathrm{C}(3)-\mathrm{C}(4)-\mathrm{C}(5)$ & $108.2(5)$ & $\mathrm{C}(29)-\mathrm{C}(30)-\mathrm{C}(31)$ & $119.9(6)$ \\
\hline $\mathrm{C}(3)-\mathrm{C}(4)-\mathrm{C}(19)$ & $127.1(5)$ & $\mathrm{C}(30)-\mathrm{C}(31)-\mathrm{C}(26)$ & $121.7(6)$ \\
\hline $\mathrm{C}(5)-\mathrm{C}(4)-\mathrm{C}(19)$ & $124.4(5)$ & $\mathrm{C}(33)-\mathrm{C}(32)-\mathrm{C}(37)$ & $119.0(5)$ \\
\hline $\mathrm{C}(3)-\mathrm{C}(4)-\mathrm{Ru}$ & $69.6(3)$ & $\mathrm{C}(33)-\mathrm{C}(32)-\mathrm{N}(1)$ & $122.1(5)$ \\
\hline$C(5)-C(4)-R u$ & $72.2(3)$ & $\mathrm{C}(37)-\mathrm{C}(32)-\mathrm{N}(1)$ & $118.9(5)$ \\
\hline$C(19)-C(4)-R u$ & $129.1(4)$ & $\mathrm{C}(32)-\mathrm{C}(33)-\mathrm{C}(34)$ & $121.1(6)$ \\
\hline $\mathrm{C}(4)-\mathrm{C}(5)-\mathrm{C}(1)$ & $108.6(5)$ & $\mathrm{C}(33)-\mathrm{C}(34)-\mathrm{C}(35)$ & $120.6(6)$ \\
\hline$C(4)-C(5)-C(26)$ & $124.1(5)$ & $\mathrm{C}(36)-\mathrm{C}(35)-\mathrm{N}(2)$ & $121.6(6)$ \\
\hline$C(1)-C(5)-C(26)$ & $123.2(5)$ & $C(36)-C(35)-C(34)$ & $117.8(6)$ \\
\hline$C(4)-C(5)-R u$ & $69.7(3)$ & $\mathrm{N}(2)-\mathrm{C}(35)-\mathrm{C}(34)$ & $120.6(6)$ \\
\hline$C(1)-C(5)-R u$ & $78.4(4)$ & $\mathrm{C}(37)-\mathrm{C}(36)-\mathrm{C}(35)$ & $120.5(6)$ \\
\hline $\mathrm{C}(26)-\mathrm{C}(5)-\mathrm{Ru}$ & $136.2(4)$ & $\mathrm{C}(36)-\mathrm{C}(37)-\mathrm{C}(32)$ & $120.9(6)$ \\
\hline$C(7)-C(6)-C(11)$ & $118.9(6)$ & N(2)-C(38)-C(39) & $110.0(6)$ \\
\hline $\mathrm{C}(7)-\mathrm{C}(6)-\mathrm{C}(2)$ & $118.3(6)$ & $\mathrm{C}(44)-\mathrm{C}(39)-\mathrm{C}(40)$ & $120.6(7)$ \\
\hline $\mathrm{C}(11)-\mathrm{C}(6)-\mathrm{C}(2)$ & $122.5(5)$ & $\mathrm{C}(44)-\mathrm{C}(39)-\mathrm{C}(38)$ & $119.9(7)$ \\
\hline $\mathrm{C}(6)-\mathrm{C}(7)-\mathrm{C}(8)$ & $120.3(6)$ & $\mathrm{C}(40)-\mathrm{C}(39)-\mathrm{C}(38)$ & $119.5(7)$ \\
\hline
\end{tabular}


S-36

$\begin{array}{llll}\mathrm{C}(39)-\mathrm{C}(40)-\mathrm{C}(41) & 119.6(7) & \mathrm{C}(39)-\mathrm{C}(44)-\mathrm{C}(43) & 121.2(7) \\ \mathrm{C}(42)-\mathrm{C}(41)-\mathrm{C}(40) & 118.3(7) & \mathrm{O}(2)-\mathrm{C}(45)-\mathrm{Ru} & 174.5(5) \\ \mathrm{C}(41)-\mathrm{C}(42)-\mathrm{C}(43) & 121.8(8) & \mathrm{O}(3)-\mathrm{C}(46)-\mathrm{Ru} & 173.4(6) \\ \mathrm{C}(42)-\mathrm{C}(43)-\mathrm{C}(44) & 118.4(8) & & \end{array}$

Symmetry transformations used to generate equivalent atoms: 
Table S7. Anisotropic displacement parameters $\left(\AA^{2} \times 10^{3}\right)$ for $\left[2,5-\mathrm{Ph}_{2}-3,4-\mathrm{Tol}_{2}\left(\eta^{4}-\right.\right.$ $\left.\left.\mathrm{C}_{4} \mathrm{CO}\right)\right](\mathrm{CO})_{2} \mathrm{RuNH}_{2} \mathrm{C}_{6} \mathrm{H}_{4}-p-\mathrm{NHCH}_{2} \mathrm{Ph}(9)$. The anisotropic displacement factor exponent takes the form: $-2 \pi^{2}\left[h^{2} a^{* 2} U^{11}+\ldots+2 h k a^{*} b^{*} U^{12}\right]$

\begin{tabular}{|c|c|c|c|c|c|c|}
\hline & $\mathrm{U}^{11}$ & $\mathrm{U}^{22}$ & $\mathrm{U}^{33}$ & $\mathrm{U}^{23}$ & $\mathrm{U}^{13}$ & $\mathrm{U}^{12}$ \\
\hline $\mathrm{Ru}$ & $17(1)$ & $25(1)$ & $21(1)$ & $1(1)$ & $2(1)$ & $-1(1)$ \\
\hline $\mathrm{O}(1)$ & $18(3)$ & $31(2)$ & $24(2)$ & $2(2)$ & $5(2)$ & $-5(2)$ \\
\hline $\mathrm{O}(2)$ & $30(3)$ & $35(3)$ & $43(3)$ & $-2(2)$ & $3(2)$ & $-3(2)$ \\
\hline $\mathrm{O}(3)$ & $28(3)$ & $27(3)$ & $45(3)$ & $1(2)$ & $9(2)$ & $3(3)$ \\
\hline $\mathrm{N}(1)$ & $18(3)$ & $24(3)$ & $28(3)$ & $-2(2)$ & $0(2)$ & $0(2)$ \\
\hline $\mathrm{N}(2)$ & $41(4)$ & $29(3)$ & $40(4)$ & $1(3)$ & $-16(3)$ & $-3(3)$ \\
\hline $\mathrm{C}(1)$ & $24(5)$ & $32(4)$ & $21(4)$ & $-9(4)$ & $0(4)$ & $0(4)$ \\
\hline $\mathrm{C}(2)$ & $11(4)$ & $26(3)$ & $24(3)$ & $-3(3)$ & $0(3)$ & $1(3)$ \\
\hline $\mathrm{C}(3)$ & $21(4)$ & $23(3)$ & $19(3)$ & $0(3)$ & $-1(3)$ & $-5(3)$ \\
\hline $\mathrm{C}(4)$ & $12(4)$ & $27(3)$ & $22(3)$ & $-3(3)$ & $2(3)$ & $0(3)$ \\
\hline $\mathrm{C}(5)$ & $19(4)$ & $24(3)$ & $20(3)$ & $3(3)$ & $-3(3)$ & $4(3)$ \\
\hline $\mathrm{C}(6)$ & $15(4)$ & $28(3)$ & $16(3)$ & $-4(3)$ & $0(3)$ & $4(3)$ \\
\hline $\mathrm{C}(7)$ & $24(4)$ & $27(3)$ & $33(4)$ & $4(3)$ & $7(3)$ & $-1(3)$ \\
\hline $\mathrm{C}(8)$ & $38(5)$ & $37(5)$ & $47(4)$ & $11(3)$ & $11(4)$ & $6(4)$ \\
\hline $\mathrm{C}(9)$ & $23(4)$ & $39(3)$ & $40(3)$ & $13(6)$ & $6(3)$ & $17(6)$ \\
\hline$C(10)$ & $25(5)$ & $57(5)$ & $27(4)$ & $13(3)$ & $6(3)$ & $10(4)$ \\
\hline $\mathrm{C}(11)$ & $28(4)$ & $25(3)$ & $26(4)$ & $4(3)$ & $3(3)$ & $2(3)$ \\
\hline $\mathrm{C}(12)$ & $19(4)$ & $31(4)$ & $17(4)$ & $-9(3)$ & $-2(3)$ & $2(3)$ \\
\hline $\mathrm{C}(13)$ & $25(4)$ & $29(5)$ & $22(3)$ & $-2(3)$ & $1(3)$ & $-6(3)$ \\
\hline $\mathrm{C}(14)$ & $30(4)$ & $35(4)$ & $28(4)$ & $2(3)$ & $6(3)$ & $-7(3)$ \\
\hline$C(15)$ & $23(4)$ & $58(4)$ & $17(4)$ & $6(3)$ & $3(3)$ & $5(3)$ \\
\hline$C(16)$ & $25(4)$ & $51(4)$ & $19(4)$ & $-3(3)$ & $3(3)$ & $6(3)$ \\
\hline $\mathrm{C}(17)$ & $28(4)$ & $39(4)$ & $23(4)$ & $-1(3)$ & $-3(3)$ & $1(3)$ \\
\hline $\mathrm{C}(18)$ & $49(5)$ & $78(7)$ & $38(4)$ & $4(4)$ & $22(3)$ & $3(4)$ \\
\hline C(19) & $15(3)$ & $20(4)$ & $27(3)$ & $4(3)$ & $2(2)$ & $-1(3)$ \\
\hline$C(20)$ & $32(5)$ & $37(4)$ & $29(4)$ & $12(3)$ & $2(3)$ & $-3(3)$ \\
\hline $\mathrm{C}(21)$ & $30(5)$ & $41(4)$ & $47(5)$ & $11(4)$ & $1(4)$ & $-2(4)$ \\
\hline$C(22)$ & $33(5)$ & $60(5)$ & $38(5)$ & $14(4)$ & $-6(4)$ & $-6(4)$ \\
\hline $\mathrm{C}(23)$ & $48(5)$ & $60(5)$ & $23(4)$ & $6(3)$ & $-11(4)$ & $-12(4)$ \\
\hline $\mathrm{C}(24)$ & $23(3)$ & $47(3)$ & $28(3)$ & $-14(6)$ & $-4(3)$ & $3(6)$ \\
\hline$C(25)$ & $49(6)$ & $94(7)$ & $58(6)$ & $33(5)$ & $-17(4)$ & $9(5)$ \\
\hline$C(26)$ & $21(4)$ & $28(3)$ & $29(4)$ & $6(3)$ & $5(3)$ & $-3(3)$ \\
\hline$C(27)$ & $25(4)$ & $31(3)$ & $26(4)$ & $1(3)$ & $0(3)$ & $-1(3)$ \\
\hline $\mathrm{C}(28)$ & $25(4)$ & $36(4)$ & $45(5)$ & $3(3)$ & $13(4)$ & $7(3)$ \\
\hline C(29) & $13(4)$ & $34(4)$ & $54(5)$ & $14(3)$ & $-2(4)$ & $-6(3)$ \\
\hline$C(30)$ & $18(4)$ & $44(4)$ & $32(4)$ & $2(3)$ & $-4(3)$ & $-2(3)$ \\
\hline
\end{tabular}


S-38

\begin{tabular}{llllccc}
$\mathrm{C}(31)$ & $15(4)$ & $46(4)$ & $34(4)$ & $0(3)$ & $-1(3)$ & $-5(3)$ \\
$\mathrm{C}(32)$ & $19(4)$ & $33(3)$ & $13(3)$ & $0(3)$ & $2(3)$ & $-2(3)$ \\
$\mathrm{C}(33)$ & $32(5)$ & $24(3)$ & $24(4)$ & $1(3)$ & $-6(3)$ & $-5(3)$ \\
$\mathrm{C}(34)$ & $39(5)$ & $21(3)$ & $38(4)$ & $2(3)$ & $1(4)$ & $2(3)$ \\
$\mathrm{C}(35)$ & $30(5)$ & $36(4)$ & $27(4)$ & $1(3)$ & $-8(3)$ & $1(4)$ \\
$\mathrm{C}(36)$ & $28(4)$ & $33(5)$ & $29(3)$ & $-10(4)$ & $-6(3)$ & $1(4)$ \\
$\mathrm{C}(37)$ & $31(4)$ & $13(4)$ & $35(4)$ & $0(3)$ & $6(3)$ & $3(3)$ \\
$\mathrm{C}(38)$ & $36(4)$ & $44(5)$ & $48(4)$ & $-4(4)$ & $-7(3)$ & $2(5)$ \\
$\mathrm{C}(39)$ & $42(5)$ & $34(4)$ & $38(5)$ & $-2(3)$ & $-2(4)$ & $1(3)$ \\
$\mathrm{C}(40)$ & $34(5)$ & $46(4)$ & $41(5)$ & $4(3)$ & $4(4)$ & $2(4)$ \\
$\mathrm{C}(41)$ & $30(5)$ & $66(5)$ & $56(5)$ & $-5(4)$ & $-1(4)$ & $2(4)$ \\
$\mathrm{C}(42)$ & $42(6)$ & $63(5)$ & $53(6)$ & $-7(4)$ & $-15(4)$ & $2(4)$ \\
$\mathrm{C}(43)$ & $61(7)$ & $87(6)$ & $38(5)$ & $-3(4)$ & $-3(5)$ & $31(5)$ \\
$\mathrm{C}(44)$ & $52(6)$ & $78(6)$ & $42(6)$ & $-6(4)$ & $14(5)$ & $14(5)$ \\
$\mathrm{C}(45)$ & $14(4)$ & $27(3)$ & $22(3)$ & $-2(3)$ & $0(3)$ & $-5(3)$ \\
$\mathrm{C}(46)$ & $23(5)$ & $42(5)$ & $14(4)$ & $8(3)$ & $2(3)$ & $-2(4)$ \\
& & & & & & \\
\hline
\end{tabular}


Table S8. Hydrogen coordinates ( x 10 $)$ and isotropic displacement parameters $\left(\AA^{2} \times 10^{3}\right)$ for $\left[2,5-\mathrm{Ph}_{2}-3,4-\mathrm{Tol}_{2}\left(\eta^{4}-\mathrm{C}_{4} \mathrm{CO}\right)\right](\mathrm{CO})_{2} \mathrm{RuNH}_{2} \mathrm{C}_{6} \mathrm{H}_{4}-p-\mathrm{NHCH}_{2} \mathrm{Ph}(\mathbf{9})$.

\begin{tabular}{|c|c|c|c|c|}
\hline & $\mathrm{x}$ & $\mathrm{y}$ & $\mathrm{z}$ & $\mathrm{U}(\mathrm{eq})$ \\
\hline $\mathrm{H}(1 \mathrm{~A})$ & 9564 & 9095 & -159 & 29 \\
\hline $\mathrm{H}(1 \mathrm{~B})$ & 9448 & 7634 & -325 & 29 \\
\hline $\mathrm{H}(2)$ & 5113 & 10147 & -2624 & 47 \\
\hline $\mathrm{H}(7)$ & 8560 & 12732 & 1203 & 33 \\
\hline $\mathrm{H}(8)$ & 7103 & 14307 & 898 & 48 \\
\hline $\mathrm{H}(9)$ & 5184 & 13636 & 641 & 41 \\
\hline $\mathrm{H}(10)$ & 4729 & 11334 & 696 & 43 \\
\hline $\mathrm{H}(11)$ & 6202 & 9755 & 1010 & 31 \\
\hline $\mathrm{H}(13)$ & 7542 & 6923 & 2490 & 31 \\
\hline $\mathrm{H}(14)$ & 6280 & 6630 & 3397 & 37 \\
\hline $\mathrm{H}(16)$ & 6132 & 10624 & 3695 & 38 \\
\hline $\mathrm{H}(17)$ & 7415 & 10956 & 2792 & 37 \\
\hline $\mathrm{H}(18 \mathrm{~A})$ & 5149 & 9257 & 4484 & 81 \\
\hline $\mathrm{H}(18 \mathrm{~B})$ & 5677 & 7780 & 4659 & 81 \\
\hline $\mathrm{H}(18 \mathrm{C})$ & 4582 & 7984 & 4010 & 81 \\
\hline $\mathrm{H}(20)$ & 11064 & 6498 & 2240 & 40 \\
\hline $\mathrm{H}(21)$ & 11938 & 5275 & 3289 & 48 \\
\hline $\mathrm{H}(23)$ & 10319 & 7521 & 4722 & 54 \\
\hline $\mathrm{H}(24)$ & 9387 & 8710 & 3668 & 40 \\
\hline $\mathrm{H}(25 \mathrm{~A})$ & 12043 & 6100 & 5225 & 104 \\
\hline $\mathrm{H}(25 \mathrm{~B})$ & 12465 & 4990 & 4659 & 104 \\
\hline $\mathrm{H}(25 \mathrm{C})$ & 11254 & 4813 & 4973 & 104 \\
\hline $\mathrm{H}(27)$ & 11675 & 8465 & 551 & 33 \\
\hline $\mathrm{H}(28)$ & 13706 & 8428 & 685 & 41 \\
\hline $\mathrm{H}(29)$ & 14785 & 8899 & 1871 & 41 \\
\hline $\mathrm{H}(30)$ & 13876 & 9578 & 2907 & 39 \\
\hline $\mathrm{H}(31)$ & 11889 & 9659 & 2779 & 38 \\
\hline $\mathrm{H}(33)$ & 7941 & 10624 & -545 & 33 \\
\hline $\mathrm{H}(34)$ & 6423 & 11024 & -1513 & 39 \\
\hline $\mathrm{H}(36)$ & 6406 & 7071 & -2102 & 37 \\
\hline $\mathrm{H}(37)$ & 7943 & 6710 & -1133 & 31 \\
\hline $\mathrm{H}(38 \mathrm{~A})$ & 4376 & 7625 & -2424 & 52 \\
\hline $\mathrm{H}(38 \mathrm{~B})$ & 5126 & 7644 & -3128 & 52 \\
\hline $\mathrm{H}(40)$ & 2537 & 8848 & -2553 & 49 \\
\hline $\mathrm{H}(41)$ & 930 & 9728 & -3391 & 61 \\
\hline $\mathrm{H}(42)$ & 1084 & 10006 & -4695 & 66 \\
\hline $\mathrm{H}(43)$ & 2799 & 9553 & -5174 & 76 \\
\hline $\mathrm{H}(44)$ & 4335 & 8623 & -4340 & 68 \\
\hline
\end{tabular}


Table S9. Torsion angles $\left[{ }^{\circ}\right]$ for $\left[2,5-\mathrm{Ph}_{2}-3,4-\mathrm{Tol}_{2}\left(\eta^{4}-\mathrm{C}_{4} \mathrm{CO}\right)\right](\mathrm{CO})_{2} \mathrm{RuNH}_{2} \mathrm{C}_{6} \mathrm{H}_{4}-p-\mathrm{NHCH}_{2} \mathrm{Ph}$ (9).

\begin{tabular}{|c|c|c|c|}
\hline $\mathrm{C}(45)-\mathrm{Ru}-\mathrm{N}(1)-\mathrm{C}(32)$ & $25.4(4)$ & $\mathrm{N}(1)-\mathrm{Ru}-\mathrm{C}(2)-\mathrm{C}(1)$ & $-58.8(4)$ \\
\hline $\mathrm{C}(46)-\mathrm{Ru}-\mathrm{N}(1)-\mathrm{C}(32)$ & $114.8(4)$ & $\mathrm{C}(4)-\mathrm{Ru}-\mathrm{C}(2)-\mathrm{C}(1)$ & $77.6(4)$ \\
\hline $\mathrm{C}(3)-\mathrm{Ru}-\mathrm{N}(1)-\mathrm{C}(32)$ & $-81.4(5)$ & $\mathrm{C}(5)-\mathrm{Ru}-\mathrm{C}(2)-\mathrm{C}(1)$ & $34.5(4)$ \\
\hline C(4)-Ru-N(1)-C(32) & $-138.6(4)$ & $\mathrm{C}(45)-\mathrm{Ru}-\mathrm{C}(2)-\mathrm{C}(6)$ & $-34.6(5)$ \\
\hline $\mathrm{C}(5)-\mathrm{Ru}-\mathrm{N}(1)-\mathrm{C}(32)$ & $-140.3(4)$ & $\mathrm{C}(46)-\mathrm{Ru}-\mathrm{C}(2)-\mathrm{C}(6)$ & $-150.6(8)$ \\
\hline $\mathrm{C}(2)-\mathrm{Ru}-\mathrm{N}(1)-\mathrm{C}(32)$ & $-76.8(4)$ & $\mathrm{C}(3)-\mathrm{Ru}-\mathrm{C}(2)-\mathrm{C}(6)$ & $-120.0(6)$ \\
\hline $\mathrm{C}(1)-\mathrm{Ru}-\mathrm{N}(1)-\mathrm{C}(32)$ & $-107.9(4)$ & $\mathrm{N}(1)-\mathrm{Ru}-\mathrm{C}(2)-\mathrm{C}(6)$ & $64.9(5)$ \\
\hline $\mathrm{C}(45)-\mathrm{Ru}-\mathrm{C}(1)-\mathrm{O}(1)$ & $-89.1(6)$ & $\mathrm{C}(4)-\mathrm{Ru}-\mathrm{C}(2)-\mathrm{C}(6)$ & $-158.7(5)$ \\
\hline $\mathrm{C}(46)-\mathrm{Ru}-\mathrm{C}(1)-\mathrm{O}(1)$ & $91.3(7)$ & $\mathrm{C}(5)-\mathrm{Ru}-\mathrm{C}(2)-\mathrm{C}(6)$ & $158.2(6)$ \\
\hline $\mathrm{C}(3)-\mathrm{Ru}-\mathrm{C}(1)-\mathrm{O}(1)$ & $-158.2(7)$ & $\mathrm{C}(1)-\mathrm{Ru}-\mathrm{C}(2)-\mathrm{C}(6)$ & $123.7(7)$ \\
\hline $\mathrm{N}(1)-\mathrm{Ru}-\mathrm{C}(1)-\mathrm{O}(1)$ & $2.2(6)$ & $\mathrm{C}(1)-\mathrm{C}(2)-\mathrm{C}(3)-\mathrm{C}(4)$ & $-2.7(6)$ \\
\hline $\mathrm{C}(4)-\mathrm{Ru}-\mathrm{C}(1)-\mathrm{O}(1)$ & $158.2(7)$ & $\mathrm{C}(6)-\mathrm{C}(2)-\mathrm{C}(3)-\mathrm{C}(4)$ & $-171.1(5)$ \\
\hline $\mathrm{C}(5)-\mathrm{Ru}-\mathrm{C}(1)-\mathrm{O}(1)$ & $118.6(8)$ & Ru-C(2)-C(3)-C(4) & $64.7(4)$ \\
\hline $\mathrm{C}(2)-\mathrm{Ru}-\mathrm{C}(1)-\mathrm{O}(1)$ & $-119.3(8)$ & $\mathrm{C}(1)-\mathrm{C}(2)-\mathrm{C}(3)-\mathrm{C}(12)$ & $165.7(6)$ \\
\hline $\mathrm{C}(45)-\mathrm{Ru}-\mathrm{C}(1)-\mathrm{C}(5)$ & $152.2(3)$ & $C(6)-C(2)-C(3)-C(12)$ & $-2.7(9)$ \\
\hline $\mathrm{C}(46)-\mathrm{Ru}-\mathrm{C}(1)-\mathrm{C}(5)$ & $-27.4(5)$ & $\mathrm{Ru}-\mathrm{C}(2)-\mathrm{C}(3)-\mathrm{C}(12)$ & $-126.9(6)$ \\
\hline $\mathrm{C}(3)-\mathrm{Ru}-\mathrm{C}(1)-\mathrm{C}(5)$ & $83.1(4)$ & $C(1)-C(2)-C(3)-R u$ & $-67.4(4)$ \\
\hline $\mathrm{N}(1)-\mathrm{Ru}-\mathrm{C}(1)-\mathrm{C}(5)$ & $-116.5(4)$ & $C(6)-C(2)-C(3)-R u$ & $124.1(6)$ \\
\hline $\mathrm{C}(4)-\mathrm{Ru}-\mathrm{C}(1)-\mathrm{C}(5)$ & $39.6(3)$ & $\mathrm{C}(45)-\mathrm{Ru}-\mathrm{C}(3)-\mathrm{C}(4)$ & $144.3(3)$ \\
\hline C(2)-Ru-C(1)-C(5) & $122.0(5)$ & $\mathrm{C}(46)-\mathrm{Ru}-\mathrm{C}(3)-\mathrm{C}(4)$ & $52.3(4)$ \\
\hline $\mathrm{C}(45)-\mathrm{Ru}-\mathrm{C}(1)-\mathrm{C}(2)$ & $30.2(5)$ & $\mathrm{N}(1)-\mathrm{Ru}-\mathrm{C}(3)-\mathrm{C}(4)$ & $-108.3(4)$ \\
\hline $\mathrm{C}(46)-\mathrm{Ru}-\mathrm{C}(1)-\mathrm{C}(2)$ & $-149.4(4)$ & $\mathrm{C}(5)-\mathrm{Ru}-\mathrm{C}(3)-\mathrm{C}(4)$ & $-37.4(3)$ \\
\hline C(3)-Ru-C(1)-C(2) & $-38.9(3)$ & $\mathrm{C}(2)-\mathrm{Ru}-\mathrm{C}(3)-\mathrm{C}(4)$ & $-115.5(4)$ \\
\hline $\mathrm{N}(1)-\mathrm{Ru}-\mathrm{C}(1)-\mathrm{C}(2)$ & $121.5(4)$ & $\mathrm{C}(1)-\mathrm{Ru}-\mathrm{C}(3)-\mathrm{C}(4)$ & $-78.4(3)$ \\
\hline $\mathrm{C}(4)-\mathrm{Ru}-\mathrm{C}(1)-\mathrm{C}(2)$ & $-82.5(4)$ & $\mathrm{C}(45)-\mathrm{Ru}-\mathrm{C}(3)-\mathrm{C}(2)$ & $-100.2(4)$ \\
\hline $\mathrm{C}(5)-\mathrm{Ru}-\mathrm{C}(1)-\mathrm{C}(2)$ & $-122.0(5)$ & $\mathrm{C}(46)-\mathrm{Ru}-\mathrm{C}(3)-\mathrm{C}(2)$ & $167.8(4)$ \\
\hline $\mathrm{O}(1)-\mathrm{C}(1)-\mathrm{C}(2)-\mathrm{C}(3)$ & $177.6(6)$ & N(1)-Ru-C(3)-C(2) & $7.2(5)$ \\
\hline $\mathrm{C}(5)-\mathrm{C}(1)-\mathrm{C}(2)-\mathrm{C}(3)$ & $7.9(7)$ & $\mathrm{C}(4)-\mathrm{Ru}-\mathrm{C}(3)-\mathrm{C}(2)$ & $115.5(4)$ \\
\hline $\mathrm{Ru}-\mathrm{C}(1)-\mathrm{C}(2)-\mathrm{C}(3)$ & $60.9(4)$ & $\mathrm{C}(5)-\mathrm{Ru}-\mathrm{C}(3)-\mathrm{C}(2)$ & $78.1(3)$ \\
\hline $\mathrm{O}(1)-\mathrm{C}(1)-\mathrm{C}(2)-\mathrm{C}(6)$ & $-13.5(10)$ & $\mathrm{C}(1)-\mathrm{Ru}-\mathrm{C}(3)-\mathrm{C}(2)$ & 37.1(3) \\
\hline$C(5)-C(1)-C(2)-C(6)$ & $176.8(5)$ & $\mathrm{C}(45)-\mathrm{Ru}-\mathrm{C}(3)-\mathrm{C}(12)$ & $23.0(6)$ \\
\hline Ru-C(1)-C(2)-C(6) & $-130.2(6)$ & $\mathrm{C}(46)-\mathrm{Ru}-\mathrm{C}(3)-\mathrm{C}(12)$ & $-68.9(6)$ \\
\hline $\mathrm{O}(1)-\mathrm{C}(1)-\mathrm{C}(2)-\mathrm{Ru}$ & $116.7(7)$ & N(1)-Ru-C(3)-C(12) & $130.5(5)$ \\
\hline$C(5)-C(1)-C(2)-R u$ & $-53.0(4)$ & $\mathrm{C}(4)-\mathrm{Ru}-\mathrm{C}(3)-\mathrm{C}(12)$ & $-121.3(6)$ \\
\hline $\mathrm{C}(45)-\mathrm{Ru}-\mathrm{C}(2)-\mathrm{C}(3)$ & $85.4(4)$ & $\mathrm{C}(5)-\mathrm{Ru}-\mathrm{C}(3)-\mathrm{C}(12)$ & $-158.6(6)$ \\
\hline $\mathrm{C}(46)-\mathrm{Ru}-\mathrm{C}(2)-\mathrm{C}(3)$ & $-30.7(9)$ & $\mathrm{C}(2)-\mathrm{Ru}-\mathrm{C}(3)-\mathrm{C}(12)$ & $123.2(7)$ \\
\hline N(1)-Ru-C(2)-C(3) & $-175.1(3)$ & $\mathrm{C}(1)-\mathrm{Ru}-\mathrm{C}(3)-\mathrm{C}(12)$ & $160.4(6)$ \\
\hline $\mathrm{C}(4)-\mathrm{Ru}-\mathrm{C}(2)-\mathrm{C}(3)$ & $-38.7(3)$ & $\mathrm{C}(2)-\mathrm{C}(3)-\mathrm{C}(4)-\mathrm{C}(5)$ & $-3.8(6)$ \\
\hline $\mathrm{C}(5)-\mathrm{Ru}-\mathrm{C}(2)-\mathrm{C}(3)$ & $-81.8(4)$ & $\mathrm{C}(12)-\mathrm{C}(3)-\mathrm{C}(4)-\mathrm{C}(5)$ & $-172.2(5)$ \\
\hline $\mathrm{C}(1)-\mathrm{Ru}-\mathrm{C}(2)-\mathrm{C}(3)$ & $-116.3(5)$ & $\mathrm{Ru}-\mathrm{C}(3)-\mathrm{C}(4)-\mathrm{C}(5)$ & $62.2(4)$ \\
\hline $\mathrm{C}(45)-\mathrm{Ru}-\mathrm{C}(2)-\mathrm{C}(1)$ & $-158.3(4)$ & $\mathrm{C}(2)-\mathrm{C}(3)-\mathrm{C}(4)-\mathrm{C}(19)$ & $169.7(5)$ \\
\hline $\mathrm{C}(46)-\mathrm{Ru}-\mathrm{C}(2)-\mathrm{C}(1)$ & $85.6(9)$ & $C(12)-C(3)-C(4)-C(19)$ & $1.3(9)$ \\
\hline C(3)-Ru-C(2)-C(1) & $116.3(5)$ & Ru-C(3)-C(4)-C(19) & $-124.2(5)$ \\
\hline
\end{tabular}




\begin{tabular}{|c|c|}
\hline$C(2)-C(3)-C(4)-R u$ & $-66.0(4)$ \\
\hline $\mathrm{C}(12)-\mathrm{C}(3)-\mathrm{C}(4)-\mathrm{Ru}$ & $125.5(6)$ \\
\hline $\mathrm{C}(45)-\mathrm{Ru}-\mathrm{C}(4)-\mathrm{C}(3)$ & $-45.3(4)$ \\
\hline $\mathrm{C}(46)-\mathrm{Ru}-\mathrm{C}(4)-\mathrm{C}(3)$ & $-138.4(4)$ \\
\hline N(1)-Ru-C(4)-C(3) & $115.3(3)$ \\
\hline $\mathrm{C}(5)-\mathrm{Ru}-\mathrm{C}(4)-\mathrm{C}(3)$ & $118.0(4)$ \\
\hline $\mathrm{C}(2)-\mathrm{Ru}-\mathrm{C}(4)-\mathrm{C}(3)$ & $38.8(3)$ \\
\hline C(1)-Ru-C(4)-C(3) & $80.2(3)$ \\
\hline $\mathrm{C}(45)-\mathrm{Ru}-\mathrm{C}(4)-\mathrm{C}(5)$ & $-163.3(3)$ \\
\hline$C(46)-R u-C(4)-C(5)$ & $103.6(4)$ \\
\hline $\mathrm{C}(3)-\mathrm{Ru}-\mathrm{C}(4)-\mathrm{C}(5)$ & $-118.0(4)$ \\
\hline $\mathrm{N}(1)-\mathrm{Ru}-\mathrm{C}(4)-\mathrm{C}(5)$ & $-2.7(4)$ \\
\hline $\mathrm{C}(2)-\mathrm{Ru}-\mathrm{C}(4)-\mathrm{C}(5)$ & $-79.2(3)$ \\
\hline $\mathrm{C}(1)-\mathrm{Ru}-\mathrm{C}(4)-\mathrm{C}(5)$ & $-37.8(3)$ \\
\hline $\mathrm{C}(45)-\mathrm{Ru}-\mathrm{C}(4)-\mathrm{C}(19)$ & $76.5(5)$ \\
\hline $\mathrm{C}(46)-\mathrm{Ru}-\mathrm{C}(4)-\mathrm{C}(19)$ & $-16.6(5)$ \\
\hline C(3)-Ru-C(4)-C(19) & $121.7(6)$ \\
\hline $\mathrm{N}(1)-\mathrm{Ru}-\mathrm{C}(4)-\mathrm{C}(19)$ & $-122.9(4)$ \\
\hline $\mathrm{C}(5)-\mathrm{Ru}-\mathrm{C}(4)-\mathrm{C}(19)$ & $-120.2(6)$ \\
\hline $\mathrm{C}(2)-\mathrm{Ru}-\mathrm{C}(4)-\mathrm{C}(19)$ & $160.6(5)$ \\
\hline $\mathrm{C}(1)-\mathrm{Ru}-\mathrm{C}(4)-\mathrm{C}(19)$ & $-158.0(6)$ \\
\hline $\mathrm{C}(3)-\mathrm{C}(4)-\mathrm{C}(5)-\mathrm{C}(1)$ & $8.9(7)$ \\
\hline$C(19)-C(4)-C(5)-C(1)$ & $-164.9(5)$ \\
\hline $\mathrm{Ru}-\mathrm{C}(4)-\mathrm{C}(5)-\mathrm{C}(1)$ & $69.5(4)$ \\
\hline$C(3)-C(4)-C(5)-C(26)$ & $166.7(5)$ \\
\hline$C(19)-C(4)-C(5)-C(26)$ & $-7.1(9)$ \\
\hline $\mathrm{Ru}-\mathrm{C}(4)-\mathrm{C}(5)-\mathrm{C}(26)$ & $-132.7(5)$ \\
\hline$C(3)-C(4)-C(5)-R u$ & $-60.6(4)$ \\
\hline $\mathrm{C}(19)-\mathrm{C}(4)-\mathrm{C}(5)-\mathrm{Ru}$ & $125.7(5)$ \\
\hline $\mathrm{O}(1)-\mathrm{C}(1)-\mathrm{C}(5)-\mathrm{C}(4)$ & $-180.0(6)$ \\
\hline$C(2)-C(1)-C(5)-C(4)$ & $-10.2(7)$ \\
\hline $\mathrm{Ru}-\mathrm{C}(1)-\mathrm{C}(5)-\mathrm{C}(4)$ & $-63.7(4)$ \\
\hline $\mathrm{O}(1)-\mathrm{C}(1)-\mathrm{C}(5)-\mathrm{C}(26)$ & $22.0(10)$ \\
\hline$C(2)-C(1)-C(5)-C(26)$ & $-168.3(5)$ \\
\hline $\mathrm{Ru}-\mathrm{C}(1)-\mathrm{C}(5)-\mathrm{C}(26)$ & $138.3(6)$ \\
\hline $\mathrm{O}(1)-\mathrm{C}(1)-\mathrm{C}(5)-\mathrm{Ru}$ & $-116.3(7)$ \\
\hline $\mathrm{C}(2)-\mathrm{C}(1)-\mathrm{C}(5)-\mathrm{Ru}$ & $53.4(4)$ \\
\hline $\mathrm{C}(45)-\mathrm{Ru}-\mathrm{C}(5)-\mathrm{C}(4)$ & $42.1(8)$ \\
\hline $\mathrm{C}(46)-\mathrm{Ru}-\mathrm{C}(5)-\mathrm{C}(4)$ & $-83.6(4)$ \\
\hline $\mathrm{C}(3)-\mathrm{Ru}-\mathrm{C}(5)-\mathrm{C}(4)$ & $37.4(3)$ \\
\hline $\mathrm{N}(1)-\mathrm{Ru}-\mathrm{C}(5)-\mathrm{C}(4)$ & 178.1(3) \\
\hline $\mathrm{C}(2)-\mathrm{Ru}-\mathrm{C}(5)-\mathrm{C}(4)$ & $80.4(3)$ \\
\hline $\mathrm{C}(1)-\mathrm{Ru}-\mathrm{C}(5)-\mathrm{C}(4)$ & $115.0(5)$ \\
\hline $\mathrm{C}(45)-\mathrm{Ru}-\mathrm{C}(5)-\mathrm{C}(1)$ & $-73.0(7)$ \\
\hline $\mathrm{C}(46)-\mathrm{Ru}-\mathrm{C}(5)-\mathrm{C}(1)$ & $161.4(3)$ \\
\hline C(3)-Ru-C(5)-C(1) & $-77.6(4)$ \\
\hline
\end{tabular}

\begin{tabular}{|c|c|}
\hline $\mathrm{N}(1)-\mathrm{Ru}-\mathrm{C}(5)-\mathrm{C}(1)$ & $63.1(4)$ \\
\hline $\mathrm{C}(4)-\mathrm{Ru}-\mathrm{C}(5)-\mathrm{C}(1)$ & $-115.0(5)$ \\
\hline $\mathrm{C}(2)-\mathrm{Ru}-\mathrm{C}(5)-\mathrm{C}(1)$ & $-34.6(3)$ \\
\hline $\mathrm{C}(45)-\mathrm{Ru}-\mathrm{C}(5)-\mathrm{C}(26)$ & $160.6(6)$ \\
\hline $\mathrm{C}(46)-\mathrm{Ru}-\mathrm{C}(5)-\mathrm{C}(26)$ & $35.0(6)$ \\
\hline C(3)-Ru-C(5)-C(26) & $155.9(6)$ \\
\hline $\mathrm{N}(1)-\mathrm{Ru}-\mathrm{C}(5)-\mathrm{C}(26)$ & $-63.4(6)$ \\
\hline$C(4)-R u-C(5)-C(26)$ & $118.5(7)$ \\
\hline$C(2)-R u-C(5)-C(26)$ & $-161.0(6)$ \\
\hline $\mathrm{C}(1)-\mathrm{Ru}-\mathrm{C}(5)-\mathrm{C}(26)$ & $-126.4(7)$ \\
\hline$C(3)-C(2)-C(6)-C(7)$ & $125.8(6)$ \\
\hline $\mathrm{C}(1)-\mathrm{C}(2)-\mathrm{C}(6)-\mathrm{C}(7)$ & $-41.1(8)$ \\
\hline $\mathrm{Ru}-\mathrm{C}(2)-\mathrm{C}(6)-\mathrm{C}(7)$ & $-143.9(5)$ \\
\hline$C(3)-C(2)-C(6)-C(11)$ & $-48.7(8)$ \\
\hline $\mathrm{C}(1)-\mathrm{C}(2)-\mathrm{C}(6)-\mathrm{C}(11)$ & $144.5(6)$ \\
\hline $\mathrm{Ru}-\mathrm{C}(2)-\mathrm{C}(6)-\mathrm{C}(11)$ & $41.7(7)$ \\
\hline $\mathrm{C}(11)-\mathrm{C}(6)-\mathrm{C}(7)-\mathrm{C}(8)$ & $0.3(9)$ \\
\hline$C(2)-C(6)-C(7)-C(8)$ & $-174.4(5)$ \\
\hline $\mathrm{C}(6)-\mathrm{C}(7)-\mathrm{C}(8)-\mathrm{C}(9)$ & $-0.3(9)$ \\
\hline $\mathrm{C}(7)-\mathrm{C}(8)-\mathrm{C}(9)-\mathrm{C}(10)$ & $0.0(8)$ \\
\hline $\mathrm{C}(8)-\mathrm{C}(9)-\mathrm{C}(10)-\mathrm{C}(11)$ & $0.2(8)$ \\
\hline$C(7)-C(6)-C(11)-C(10)$ & $-0.1(8)$ \\
\hline$C(2)-C(6)-C(11)-C(10)$ & $174.4(5)$ \\
\hline$C(9)-C(10)-C(11)-C(6)$ & $-0.1(8)$ \\
\hline$C(4)-C(3)-C(12)-C(17)$ & $116.3(7)$ \\
\hline $\mathrm{C}(2)-\mathrm{C}(3)-\mathrm{C}(12)-\mathrm{C}(17)$ & $-50.1(9)$ \\
\hline Ru-C(3)-C(12)-C(17) & $-148.5(5)$ \\
\hline $\mathrm{C}(4)-\mathrm{C}(3)-\mathrm{C}(12)-\mathrm{C}(13)$ & $-56.7(9)$ \\
\hline $\mathrm{C}(2)-\mathrm{C}(3)-\mathrm{C}(12)-\mathrm{C}(13)$ & $136.8(6)$ \\
\hline $\mathrm{Ru}-\mathrm{C}(3)-\mathrm{C}(12)-\mathrm{C}(13)$ & $38.4(9)$ \\
\hline$C(17)-C(12)-C(13)-C(14)$ & $-0.2(10)$ \\
\hline $\mathrm{C}(3)-\mathrm{C}(12)-\mathrm{C}(13)-\mathrm{C}(14)$ & $173.0(5)$ \\
\hline$C(12)-C(13)-C(14)-C(15)$ & $-0.5(10)$ \\
\hline$C(13)-C(14)-C(15)-C(16)$ & $1.3(10)$ \\
\hline $\mathrm{C}(13)-\mathrm{C}(14)-\mathrm{C}(15)-\mathrm{C}(18)$ & $-178.3(6)$ \\
\hline$C(14)-C(15)-C(16)-C(17)$ & $-1.6(10)$ \\
\hline$C(18)-C(15)-C(16)-C(17)$ & $178.0(6)$ \\
\hline $\mathrm{C}(13)-\mathrm{C}(12)-\mathrm{C}(17)-\mathrm{C}(16)$ & $-0.1(10)$ \\
\hline $\mathrm{C}(3)-\mathrm{C}(12)-\mathrm{C}(17)-\mathrm{C}(16)$ & $-173.2(6)$ \\
\hline$C(15)-C(16)-C(17)-C(12)$ & $1.1(10)$ \\
\hline $\mathrm{C}(3)-\mathrm{C}(4)-\mathrm{C}(19)-\mathrm{C}(24)$ & $-41.7(9)$ \\
\hline$C(5)-C(4)-C(19)-C(24)$ & $130.8(6)$ \\
\hline $\mathrm{Ru}-\mathrm{C}(4)-\mathrm{C}(19)-\mathrm{C}(24)$ & $-134.6(5)$ \\
\hline $\mathrm{C}(3)-\mathrm{C}(4)-\mathrm{C}(19)-\mathrm{C}(20)$ & $144.5(6)$ \\
\hline $\mathrm{C}(5)-\mathrm{C}(4)-\mathrm{C}(19)-\mathrm{C}(20)$ & $-43.0(8)$ \\
\hline $\mathrm{Ru}-\mathrm{C}(4)-\mathrm{C}(19)-\mathrm{C}(20)$ & $51.6(8)$ \\
\hline
\end{tabular}




\begin{tabular}{|c|c|c|c|}
\hline $\mathrm{C}(24)-\mathrm{C}(19)-\mathrm{C}(20)-\mathrm{C}(21)$ & $0.6(9)$ & $\mathrm{C}(40)-\mathrm{C}(39)-\mathrm{C}(44)-\mathrm{C}(43)$ & $4.2(11)$ \\
\hline$C(4)-C(19)-C(20)-C(21)$ & $174.6(6)$ & $\mathrm{C}(38)-\mathrm{C}(39)-\mathrm{C}(44)-\mathrm{C}(43)$ & $-177.4(7)$ \\
\hline $\mathrm{C}(19)-\mathrm{C}(20)-\mathrm{C}(21)-\mathrm{C}(22)$ & $-0.1(10)$ & $\mathrm{C}(42)-\mathrm{C}(43)-\mathrm{C}(44)-\mathrm{C}(39)$ & $-3.8(12)$ \\
\hline $\mathrm{C}(20)-\mathrm{C}(21)-\mathrm{C}(22)-\mathrm{C}(23)$ & $-1.6(10)$ & $\mathrm{C}(46)-\mathrm{Ru}-\mathrm{C}(45)-\mathrm{O}(2)$ & $95(5)$ \\
\hline $\mathrm{C}(20)-\mathrm{C}(21)-\mathrm{C}(22)-\mathrm{C}(25)$ & $-178.4(7)$ & $\mathrm{C}(3)-\mathrm{Ru}-\mathrm{C}(45)-\mathrm{O}(2)$ & $-29(5)$ \\
\hline $\mathrm{C}(21)-\mathrm{C}(22)-\mathrm{C}(23)-\mathrm{C}(24)$ & $2.7(11)$ & $\mathrm{N}(1)-\mathrm{Ru}-\mathrm{C}(45)-\mathrm{O}(2)$ & $-169(5)$ \\
\hline $\mathrm{C}(25)-\mathrm{C}(22)-\mathrm{C}(23)-\mathrm{C}(24)$ & 179.6(7) & $\mathrm{C}(4)-\mathrm{Ru}-\mathrm{C}(45)-\mathrm{O}(2)$ & $-3(5)$ \\
\hline $\mathrm{C}(20)-\mathrm{C}(19)-\mathrm{C}(24)-\mathrm{C}(23)$ & $0.6(10)$ & $\mathrm{C}(5)-\mathrm{Ru}-\mathrm{C}(45)-\mathrm{O}(2)$ & $-33(6)$ \\
\hline $\mathrm{C}(4)-\mathrm{C}(19)-\mathrm{C}(24)-\mathrm{C}(23)$ & $-173.4(6)$ & $\mathrm{C}(2)-\mathrm{Ru}-\mathrm{C}(45)-\mathrm{O}(2)$ & $-67(5)$ \\
\hline $\mathrm{C}(22)-\mathrm{C}(23)-\mathrm{C}(24)-\mathrm{C}(19)$ & $-2.2(11)$ & $\mathrm{C}(1)-\mathrm{Ru}-\mathrm{C}(45)-\mathrm{O}(2)$ & $-85(5)$ \\
\hline$C(4)-C(5)-C(26)-C(27)$ & $135.0(6)$ & $\mathrm{C}(45)-\mathrm{Ru}-\mathrm{C}(46)-\mathrm{O}(3)$ & $-66(7)$ \\
\hline $\mathrm{C}(1)-\mathrm{C}(5)-\mathrm{C}(26)-\mathrm{C}(27)$ & $-70.4(8)$ & $\mathrm{C}(3)-\mathrm{Ru}-\mathrm{C}(46)-\mathrm{O}(3)$ & $29(7)$ \\
\hline $\mathrm{Ru}-\mathrm{C}(5)-\mathrm{C}(26)-\mathrm{C}(27)$ & $39.3(8)$ & $\mathrm{N}(1)-\mathrm{Ru}-\mathrm{C}(46)-\mathrm{O}(3)$ & $-163(6)$ \\
\hline$C(4)-C(5)-C(26)-C(31)$ & $-48.8(8)$ & $\mathrm{C}(4)-\mathrm{Ru}-\mathrm{C}(46)-\mathrm{O}(3)$ & $59(7)$ \\
\hline$C(1)-C(5)-C(26)-C(31)$ & $105.8(7)$ & $\mathrm{C}(5)-\mathrm{Ru}-\mathrm{C}(46)-\mathrm{O}(3)$ & $97(7)$ \\
\hline $\mathrm{Ru}-\mathrm{C}(5)-\mathrm{C}(26)-\mathrm{C}(31)$ & $-144.6(5)$ & $\mathrm{C}(2)-\mathrm{Ru}-\mathrm{C}(46)-\mathrm{O}(3)$ & $52(7)$ \\
\hline $\mathrm{C}(31)-\mathrm{C}(26)-\mathrm{C}(27)-\mathrm{C}(28)$ & $1.3(8)$ & $\mathrm{C}(1)-\mathrm{Ru}-\mathrm{C}(46)-\mathrm{O}(3)$ & $114(6)$ \\
\hline $\mathrm{C}(5)-\mathrm{C}(26)-\mathrm{C}(27)-\mathrm{C}(28)$ & $177.5(5)$ & & \\
\hline $\mathrm{C}(26)-\mathrm{C}(27)-\mathrm{C}(28)-\mathrm{C}(29)$ & $1.0(8)$ & & \\
\hline C(27)-C(28)-C(29)-C(30) & $-2.6(9)$ & & \\
\hline $\mathrm{C}(28)-\mathrm{C}(29)-\mathrm{C}(30)-\mathrm{C}(31)$ & $2.0(9)$ & & \\
\hline $\mathrm{C}(29)-\mathrm{C}(30)-\mathrm{C}(31)-\mathrm{C}(26)$ & $0.3(9)$ & & \\
\hline $\mathrm{C}(27)-\mathrm{C}(26)-\mathrm{C}(31)-\mathrm{C}(30)$ & $-2.0(9)$ & & \\
\hline $\mathrm{C}(5)-\mathrm{C}(26)-\mathrm{C}(31)-\mathrm{C}(30)$ & $-178.3(5)$ & & \\
\hline $\mathrm{Ru}-\mathrm{N}(1)-\mathrm{C}(32)-\mathrm{C}(33)$ & $83.2(6)$ & & \\
\hline Ru-N(1)-C(32)-C(37) & $-96.2(6)$ & & \\
\hline $\mathrm{C}(37)-\mathrm{C}(32)-\mathrm{C}(33)-\mathrm{C}(34)$ & $-1.4(9)$ & & \\
\hline $\mathrm{N}(1)-\mathrm{C}(32)-\mathrm{C}(33)-\mathrm{C}(34)$ & $179.3(5)$ & & \\
\hline C(32)-C(33)-C(34)-C(35) & $2.0(10)$ & & \\
\hline $\mathrm{C}(38)-\mathrm{N}(2)-\mathrm{C}(35)-\mathrm{C}(36)$ & $15.7(9)$ & & \\
\hline $\mathrm{C}(38)-\mathrm{N}(2)-\mathrm{C}(35)-\mathrm{C}(34)$ & $-166.2(6)$ & & \\
\hline $\mathrm{C}(33)-\mathrm{C}(34)-\mathrm{C}(35)-\mathrm{C}(36)$ & $-2.1(10)$ & & \\
\hline $\mathrm{C}(33)-\mathrm{C}(34)-\mathrm{C}(35)-\mathrm{N}(2)$ & $179.7(6)$ & & \\
\hline $\mathrm{N}(2)-\mathrm{C}(35)-\mathrm{C}(36)-\mathrm{C}(37)$ & $179.9(6)$ & & \\
\hline $\mathrm{C}(34)-\mathrm{C}(35)-\mathrm{C}(36)-\mathrm{C}(37)$ & $1.8(9)$ & & \\
\hline $\mathrm{C}(35)-\mathrm{C}(36)-\mathrm{C}(37)-\mathrm{C}(32)$ & $-1.2(9)$ & & \\
\hline $\mathrm{C}(33)-\mathrm{C}(32)-\mathrm{C}(37)-\mathrm{C}(36)$ & $1.0(9)$ & & \\
\hline $\mathrm{N}(1)-\mathrm{C}(32)-\mathrm{C}(37)-\mathrm{C}(36)$ & $-179.6(5)$ & & \\
\hline $\mathrm{C}(35)-\mathrm{N}(2)-\mathrm{C}(38)-\mathrm{C}(39)$ & $173.7(6)$ & & \\
\hline $\mathrm{N}(2)-\mathrm{C}(38)-\mathrm{C}(39)-\mathrm{C}(44)$ & $94.6(7)$ & & \\
\hline N(2)-C(38)-C(39)-C(40) & $-87.0(7)$ & & \\
\hline $\mathrm{C}(44)-\mathrm{C}(39)-\mathrm{C}(40)-\mathrm{C}(41)$ & $-3.0(10)$ & & \\
\hline C(38)-C(39)-C(40)-C(41) & $178.6(6)$ & & \\
\hline $\mathrm{C}(39)-\mathrm{C}(40)-\mathrm{C}(41)-\mathrm{C}(42)$ & $1.5(10)$ & & \\
\hline $\mathrm{C}(40)-\mathrm{C}(41)-\mathrm{C}(42)-\mathrm{C}(43)$ & $-1.3(11)$ & & \\
\hline $\mathrm{C}(41)-\mathrm{C}(42)-\mathrm{C}(43)-\mathrm{C}(44)$ & $2.4(12)$ & & \\
\hline
\end{tabular}


Table S10. Hydrogen bonds for $\left[2,5-\mathrm{Ph}_{2}-3,4-\mathrm{Tol}_{2}\left(\eta^{4}-\mathrm{C}_{4} \mathrm{CO}\right)\right](\mathrm{CO})_{2} \mathrm{RuNH}_{2} \mathrm{C}_{6} \mathrm{H}_{4}-p-\mathrm{NHCH}_{2} \mathrm{Ph}(\mathbf{9})$ $\left[\AA ̊\right.$ and $\left.{ }^{\circ}\right]$.

\begin{tabular}{lcccc}
\hline $\mathrm{D}-\mathrm{H} \ldots \mathrm{A}$ & $\mathrm{d}(\mathrm{D}-\mathrm{H})$ & $\mathrm{d}(\mathrm{H} \ldots \mathrm{A})$ & $\mathrm{d}(\mathrm{D} \ldots \mathrm{A})$ & $<(\mathrm{DHA})$ \\
\hline $\mathrm{N}(1)-\mathrm{H}(1 \mathrm{~A}) \ldots \mathrm{O}(1)$ & 0.92 & 2.29 & $2.999(6)$ & 133.6 \\
$\mathrm{~N}(1)-\mathrm{H}(1 \mathrm{~B}) \ldots \mathrm{O}(1) \# 1$ & 0.92 & 1.95 & $2.861(5)$ & 172.1
\end{tabular}

Symmetry transformations used to generate equivalent atoms:

$\# 1-\mathrm{x}+2, \mathrm{y}-1 / 2,-\mathrm{z}$

\section{References}

(1) El-Shafei, A. K.; Abdel-Ghany, H. A. J. Indian Chem. Soc. 1984, 61, 62.

(2) Kwong, F. Y.; Klapars, A; Buchwald, S. L. Org. Lett. 2002, 4, 581.

(3) Casey, C. P.; Singer, S. W.; Powell, D. R.; Hayashi, R. K.; Kavana, M. J. Am. Chem. Soc. 2001, 123, 1090.

(4) Casey, C. P.; Bikzhanova, G. A.; Bäckvall, Jan-E.; Johansson, L.; Park, J.; Kim, Y. H. Organometallics 2002, 21, 1955-1959.

(5) (a) Becke, A. D. Phys. Rev. A 1998, 38, 3098 (b) Lee, C.; Yang, W.; Parr, R. G. Phys. Rev. B 1988, 37, 785 (c) Becke, A. D. J. Chem. Phys. 1993, 98, 5648

(6) Hay, P. J.; Wadt, W. R. J. Chem. Phys. 1985, 82, 299

(7) (a) Ditchfield, R.; Hehre, W. J.; Pople, J. A. J. Chem. Phys. 1971, 54, 724 (b) Hehre, W. J.; Ditchfield, R.; Pople, J. A. J. Chem. Phys. 1972, 56, 2257 (c) Hariharan, P. C.; Pople, J. A. Theor. Chim. Acta. 1973, 28, 213

(8) (a) Miertus, S.; Scrocco, E. and Tomasi, J. Chem. Phys . 1981, 55, 117 (b) Cammi, R.; Tomasi, J. J. Comp. Chem. 1995, 16, 1449 (c) Mennucci, B.; Cancès, E. and Tomasi, J. J. 
Phys. Chem. B 1997, 101, 10506 (d) Cancès, E.; Mennucci, B. and Tomasi, J. J. Chem. Phys. 1997, 107, 3032

(9) Krishnan, R.; Binkley, J. S.; Seeger, R.; Pople, J. A. J. Chem. Phys. 1980, 72, 650

(10) Gaussian 98, Revision A.6, M. J. Frisch, G. W. Trucks, H. B. Schlegel, G. E.

Scuseria, M. A. Robb, J. R. Cheeseman, V. G. Zakrzewski, J. A. Montgomery, Jr., R. E.

Stratmann, J. C. Burant, S. Dapprich, J. M. Millam, A. D. Daniels, K. N. Kudin, M. C. Strain, O. Farkas, J. Tomasi, V. Barone, M. Cossi, R. Cammi, B. Mennucci, C. Pomelli, C. Adamo, S. Clifford, J. Ochterski, G. A. Petersson, P. Y. Ayala, Q. Cui, K. Morokuma, D. K. Malick, A. D. Rabuck, K. Raghavachari, J. B. Foresman, J. Cioslowski, J. V. Ortiz, B. B. Stefanov, G. Liu, A. Liashenko, P. Piskorz, I. Komaromi, R. Gomperts, R. L. Martin, D. J. Fox, T. Keith, M. A. AlLaham, C. Y. Peng, A. Nanayakkara, C. Gonzalez, M. Challacombe, P. M. W. Gill, B. Johnson, W. Chen, M. W. Wong, J. L. Andres, C. Gonzalez, M. Head-Gordon, E. S. Replogle, and J. A. Pople, Gaussian, Inc., Pittsburgh PA, 1998.

(11) Bruker-AXS. (2000-2003) SADABS V.2.05, SAINT V.6.22, SHELXTL V.6.10 \& SMART 5.622 Software Reference Manuals. Bruker-AXS, Madison, Wisconsin, USA. 